\title{
AN EXTENSIBLE EQUALITY CHECKING ALGORITHM FOR DEPENDENT TYPE THEORIES
}

\author{
ANDREJ BAUER AND ANJA PETKOVIĆ KOMEL
}

Faculty of Mathematics and Physics, University of Ljubljana

e-mail address: Andrej.Bauer@andrej.com, Anja.Petkovic@fmf.uni-lj.si

\begin{abstract}
We present a general and user-extensible equality checking algorithm that is applicable to a large class of type theories. The algorithm has a type-directed phase for applying extensionality rules and a normalization phase based on computation rules, where both kinds of rules are defined using the type-theoretic concept of object-invertible rules. We also give sufficient syntactic criteria for recognizing such rules, as well as a simple pattern-matching algorithm for applying them. A third component of the algorithm is a suitable notion of principal arguments, which determines a notion of normal form. By varying these, we obtain known notions, such as weak head-normal and strong normal forms. We prove that our algorithm is sound. We implemented it in the Andromeda 2 proof assistant, which supports user-definable type theories. The user need only provide the equality rules they wish to use, which the algorithm automatically classifies as computation or extensionality rules, and select appropriate principal arguments.
\end{abstract}

\section{INTRODUCTION}

Equality checking algorithms are essential components of proof assistants based on type theories [Coq, Agd, dMKA ${ }^{+} 15, \mathrm{SBF}^{+} 19, \mathrm{GCST19}$, AOV17]. They free users from the burden of proving scores of mostly trivial judgemental equalities, and provide computationby-normalization engines. Some systems [Ded, CA16] go further by allowing user extensions to the built-in equality checkers.

The situation is less pleasant in a proof assistant that supports arbitrary user-definable theories, such as Andromeda 2 [And, $\left.\mathrm{BGH}^{+} 18\right]$, where in general no equality checking algorithm may be available. Nevertheless, the proof assistant should still provide support for equality checking that is easy to use and works well in the common, well-behaved cases. For this purpose we have developed and implemented a sound and extensible equality checking algorithm for user-definable type theories.

The generality of type theories supported by Andromeda 2 presents a significant challenge in devising a useful equality checking algorithm. Many commonly used ideas and notions that one encounters in specific type theories do not apply anymore: not every rule can be classified either as an introduction or an elimination form, not every equation as either a $\beta$ or an $\eta$-rule, all terms must be fully annotated with types to ensure soundness, there may be no reasonable notion of normal form, or neutral form, etc. And of course, the user may

Key words and phrases: type theory, equality checking, proof assistant.

(c) A. Bauer and A. Petković Komel (c) Creative Commons 
easily define a theory whose equality checking is undecidable. In order to do better than just exhaustive proof search, some compromises must therefore be made and design decisions taken:

(1) We work in the fully general setting of standard type theories (Section 2).

(2) We prefer ease of experimentation at the expense of possible non-termination or unpredictable behavior.

(3) At the same time, soundness of the algorithm is paramount: any equation verified by it must be derivable in the theory at hand.

(4) The algorithm should work well on well-behaved theories, and especially those seen in practice.

The most prominent design goals missing from the above list are completeness and performance. The former cannot be achieved in full generality, as there are type theories with undecidable equality checking. We have expended enough energy looking for acceptably general sufficient conditions guaranteeing completeness to state with confidence that this task is best left for another occasion. Regarding performance, we freely admit that equality checking in Andromeda 2 is nowhere near the efficiency of established proof assistants. For this we blame not only the immaturity of the implementation, but also the generality of the situation, which simply demands that a price be paid in exchange for soundness. We console ourselves with the fact that our equality checker achieves soundness and complete user-extensibility at the same time.

Contributions. We present a general equality checking algorithm that is applicable to a large class of type theories, the standard type theories of [HB21] (Section 2). The algorithm (Section 3.4) is fashioned after equality checking algorithms [SH06, AS12] that have a typedirected phase for applying extensionality rules (inter-derivable with $\eta$-rules), intertwined with a normalization phase based on computation rules ( $\beta$-rules). For the usual kinds of type theories (simply typed $\lambda$-calculus, Martin-Löf type theory, System F), the algorithm behaves like the well-known standard equality checkers. We prove that our algorithm is sound (Section 3.5).

We define a general notion of computation and extensionality rules (Section 3.2), using the type-theoretic concept of an object-invertible rule (Section 3.1). We also provide sufficient syntactic criteria for recognizing such rules, together with a simple pattern-matching algorithm for applying them. A third component of the algorithm is a suitable notion of normal form, which guarantees correct execution of normalization and coherent interaction of both phases of the algorithm. In our setting, normal forms are determined by a selection of principal arguments (Section 3.3). By varying these, we obtain known notions, such as weak headnormal and strong normal forms.

We implemented the algorithm in Andromeda 2 (Section 5). The user need only provide the equality rules they wish to use, which the algorithm automatically classifies either as computation or extensionality rules, rejects those that are of neither kind, and selects appropriate principal arguments.

Those readers who prefer to see examples before the formal development, may first take a peek at Section 6, where we show how our work allows one to implement extensional type theory, and use the reflection rule to derive computation rules which are only available in their propositional form in intensional type theory. 
Acknowledgments. We thank Philipp G. Haselwarter for his support and discussions through which he generously shared ideas that helped get this work completed. This material is based upon work supported by the U.S. Air Force Office of Scientific Research under award number FA9550-17-1-0326, grant number 12595060, and award number FA9550-21-1-0024.

\section{Finitary type theORIES}

We shall work with a variant of general dependent type theories [BHL20], namely finitary type theories, as described in [HB21] and implemented in Andromeda 2. We give here only an overview of the syntax of such theories and refer the reader to [HB21] for a complete exposition.

2.1. Deductive systems. We first recall the general notion of a deductive system. A (finitary) closure rule on a carrier set $S$ is a pair $\left(\left[p_{1}, \ldots, p_{n}\right], q\right)$ where $p_{1}, \ldots, p_{n}, q \in S$. The elements $p_{1}, \ldots, p_{n}$ are the premises and $q$ is the conclusion of the rule. A rule may be displayed as

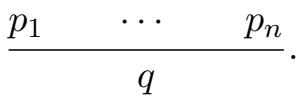

A deductive system on a set $S$ is a family $C$ of closure rules on $S$. We say that $T \subseteq S$ is deductively closed for $C$ when the following holds: for every rule $C_{i}=\left(\left[p_{1}, \ldots, p_{n}\right], q\right)$, if $\left\{p_{1}, \ldots, p_{n}\right\} \subseteq T$ then $q \in T$. A derivation with conclusion $q \in S$ is a well-founded tree whose root is labeled by an index $i$ of a closure rule $C_{i}=\left(\left[p_{1}, \ldots, p_{n}\right], q\right)$, and whose subtrees are derivations with conclusions $p_{1}, \ldots, p_{n}$. We say that $q \in S$ is derivable if there exists a derivation with conclusion $q$. The derivable elements of $S$ form precisely the least deductively closed subset.

All deductive systems that we shall consider will have as their carriers the set of hypothetical judgements and boundaries, as described in Section 2.4.

2.2. Signatures and arities. In a finitary type theory there are four judgement forms:

- "A type" asserting that $A$ is a type,

- " $t: A$ " asserting that $t$ is a term of type $A$,

- " $A \equiv B$ by $\star_{\text {Ty }}$ " asserting that types $A$ and $B$ are equal, and

- " $s \equiv t: A$ by $\star_{\mathrm{Tm}}$ " asserting that terms $s$ and $t$ are equal at type $A$.

We indicate these with tokens Ty, Tm, EqTy and EqTm respectively. To each token there also corresponds a syntactic class. Expressions of class Ty are the type expressions, and those of class $\mathrm{Tm}$ are the term expressions. These are formed using (primitive) symbols and metavariables, see Section 2.3, each of which has an associated arity, as explained below. The symbols should be thought of as the primitive type and term formers, while the metavariables shall be used to refer to the premises of a rule, and as pattern variables in the equality checking algorithm. The only expressions of syntactic classes EqTy and EqTm are the dummy

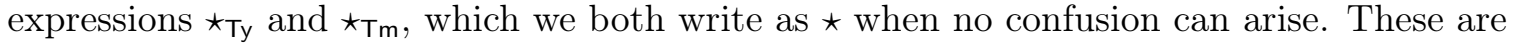
formality, to be used where one would normally record a proof term witnessing a premise, but the premise is a judgemental equality, which is proof irrelevant.

The symbol arity $\left(c,\left[\left(c_{1}, n_{1}\right), \ldots,\left(c_{k}, n_{k}\right)\right]\right)$ of a symbol $\mathrm{S}$ tells us that

(1) the syntactic class of expressions built with $\mathrm{S}$ is $c \in\{\mathrm{Ty}, \mathrm{Tm}\}$,

(2) $\mathrm{S}$ accepts $k \in \mathbb{N}$ arguments, 
(3) the $i$-th argument has syntactic class $c_{i} \in\{$ Ty, Tm, EqTy, EqTm $\}$ and binds $n_{i} \in \mathbb{N}$ variables.

Example 2.1. The arity of a type constant such as bool is (Ty, []), the arity of a binary term operation such as + is $(\mathrm{Tm},[(\mathrm{Tm}, 0),(\mathrm{Tm}, 0)])$, and the arity of a quantifier such as the dependent product $\Pi$ is $(\mathrm{Ty},[(\mathrm{Ty}, 0),(\mathrm{Ty}, 1)])$ because it is a type former taking two type arguments, with the second one binding one variable.

The metavariable arity of a metavariable $\mathrm{M}$ is a pair $(c, n)$, where the syntactic class $c \in\{\mathrm{Ty}, \mathrm{Tm}, \mathrm{EqTy}, \mathrm{EqTm}\}$ indicates whether $\mathrm{M}$ is respectively a type, term, type equality, or term equality metavariable, and $n \in \mathbb{N}$ is the number of term arguments it accepts. The metavariables of syntactic classes Ty and Tm are the object metavariables, and they participate in formation of expressions, while those of syntactic classes EqTy and EqTm are the equality metavariables, and are used to refer to equational premises.

The information about symbol arities is collected in a signature, which maps each symbol to its arity. When discussing syntax, it is understood that such a signature has been given, even if we do not mention it explicitly.

2.3. Expressions. The syntax of finitary type theories is summarized in the top part of Figure 1. There are three kinds: type expressions, term expressions, and arguments.

A type expression is an application $\mathrm{S}\left(e_{1}, \ldots, e_{n}\right)$ of a primitive symbol $\mathrm{S}$ to arguments, or an application $\mathrm{M}\left(t_{1}, \ldots, t_{n}\right)$ of a metavariable $\mathrm{M}$ to terms. We write $\mathrm{S}$ and $\mathrm{M}$ instead of $\mathrm{S}()$ and $\mathrm{M}()$.

A term expression is a variable, an application of a primitive symbol to arguments, or an application of a metavariable to terms. We strictly separate free variables $a, b, c, \ldots$ from the bound ones $x, y, z, \ldots$, a choice fashioned after the locally nameless syntax [MP93, Cha12], a common implementation technique in which free variables are represented as names and the bound ones as de Bruijn indices.

An argument is a type expression, a term expression, a dummy argument $\star_{\mathrm{T}_{\mathrm{y}}}$ or $\star_{\mathrm{T}_{\mathrm{m}}}$, or an abstracted argument $\{x\} e$ binding $x$ in $e$. Note that we take abstraction to be a basic syntactic operation. For instance, we do not construe a $\lambda$-abstraction as a variable-binding construct $\lambda x: A$.t but rather an application $\lambda(A,\{x\} t)$ of the primitive symbol $\lambda$ to two separate arguments $A$ and $\{x\} t$. We may abbreviate an iterated abstraction $\left\{x_{1}\right\} \cdots\left\{x_{n}\right\} e$ as $\{\vec{x}\} e$, and similarly use the vector notation elsewhere when appropriate. We permit $\vec{x}$ to be empty, in which case $\{\vec{x}\} e$ is just $e$. To an argument we assign the metavariable arity

$$
\operatorname{ar}\left(\left\{x_{1}\right\} \cdots\left\{x_{n}\right\} e\right)=(c, n),
$$

where $c \in\{\mathrm{Ty}, \mathrm{Tm}, \mathrm{EqTy}, \mathrm{EqTm}\}$ is the syntactic class of the non-abstracted argument $e$.

For an expression to be syntactically valid, all bound variables must be bound by abstractions, and all symbol and metavariable applications respect their arities. That is, if the arity of $\mathrm{S}$ is $\left(c,\left[\left(c_{1}, n_{1}\right), \ldots,\left(c_{k}, n_{k}\right)\right]\right)$ then it must be applied to $k$ arguments $e_{1}, \ldots, e_{k}$ with $\operatorname{ar}\left(e_{i}\right)=\left(c_{i}, n_{i}\right)$, and the expression $\mathbf{S}\left(e_{1}, \ldots, e_{k}\right)$ has syntactic class $c$. Similarly, an object metavariable $\mathrm{M}$ of arity $(c, n)$ must be applied to $n$ term expressions to yield an expression of syntactic class $c$.

We write $e[t / x]$ for capture-avoiding substitution of $t$ for $x$ in $e$, and $e\left[t_{1} / x_{1}, \ldots, t_{n} / x_{n}\right]$ or $e[\vec{t} / \vec{x}]$ for simultaneous substitution of $t_{1}, \ldots, t_{n}$ for $x_{1}, \ldots, x_{n}$. Expressions which only differ in the choice of names of bound variables are considered syntactically identical (alternatively, we could use de Bruijn indices for bound variables). 


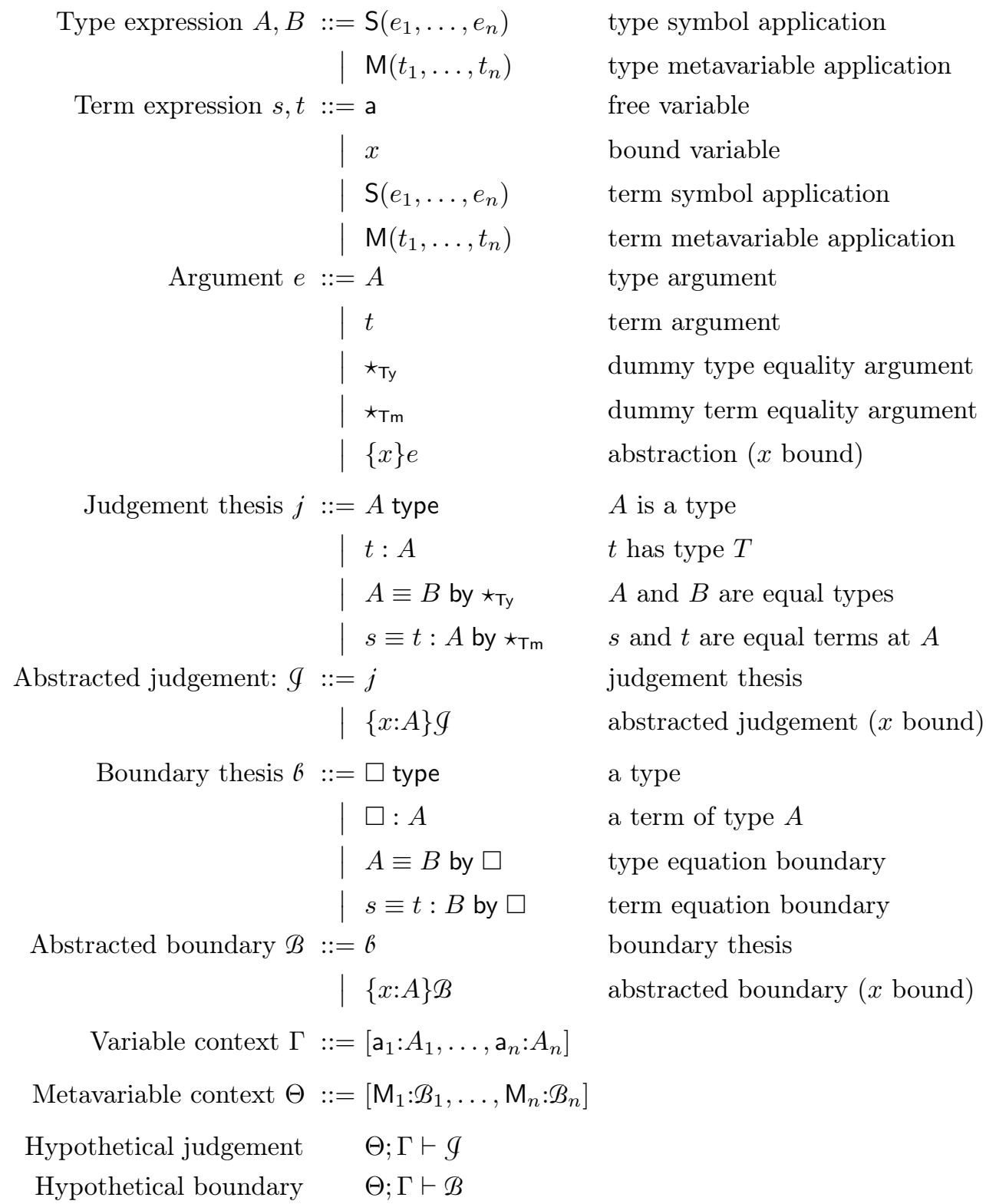

Figure 1: The syntax of expressions, boundaries and judgements.

Given an expression $e$, let $\operatorname{mv}(e)$ and $\mathrm{fv}(e)$ be the sets of metavariables and free variables occurring in $e$, respectively. A renaming of an expression $e$ is an injective map $\rho$ with domain $\operatorname{mv}(e) \cup \mathrm{fv}(e)$ that takes metavariables to metavariables and free variables to free variables. The renaming acts on $e$ to yield an expression $\rho_{*} e$ by replacing each occurrence of a metavariable $\mathbf{M}$ and a free variable a with $\rho(\mathbf{M})$ and $\rho(\mathrm{a})$, respectively. We similarly define renamings of metavariable and variable contexts, judgements, and boundaries, which are defined below. 
2.4. Judgements and boundaries. We next discuss the syntax of judgements and boundaries, see the bottom part of Figure 1. To each of the judgement forms corresponds a judgement thesis:

- "A type" asserts that $A$ is a type,

- " $t: A$ " that $t$ is a term of type $A$,

- " $A \equiv B$ by $\star_{\mathrm{Ty}}$ " that types $A$ and $B$ are equal, and

- " $s \equiv t: A$ by ${ }^{{ }_{T}} \mathrm{Tm}_{\mathrm{m}}$ " that terms $s$ and $t$ of type $A$ are equal.

The latter two have " by *" attached so that all boundaries can be filled with a head, as we shall explain shortly. We normally write just " $A \equiv B$ " and " $s \equiv t: A$ ".

A boundary is a fundamental notion of type theory, although perhaps less familiar. Whereas a judgement is an assertion, a boundary is a goal to be accomplished:

- " $\square$ type" asks that a type be constructed,

- " $\square$ : A" that the type $A$ be inhabited, and

- " $A \equiv B$ by $\square$ " and " $s \equiv t: A$ by $\square$ " that equations be proved.

An abstracted judgement has the form $\{x: A\} \mathscr{I}$, where $A$ is a type expression and $\mathscr{I}$ is a (possibly abstracted) judgement. The variable $x$ is bound in $\mathscr{I}$ but not in $A$. As before, we write $\{\vec{x}: \vec{A}\} j$ for an iterated abstraction $\left\{x_{1}: A_{1}\right\} \cdots\left\{x_{n}: A_{n}\right\} j$. Similarly, an abstracted boundary has the form $\left\{x_{1}: A_{1}\right\} \cdots\left\{x_{n}: A_{n}\right\} \mathfrak{b}$, where $\boldsymbol{b}$ is a boundary thesis, i.e., it takes one of the four (non-abstracted) boundary forms.

To an abstracted boundary we assign a metavariable arity by

$$
\operatorname{ar}\left(\left\{x_{1}: A_{1}\right\} \cdots\left\{x_{n}: A_{n}\right\} \mathscr{B}\right)=(c, n)
$$

where $c \in\{\mathrm{Ty}, \mathrm{Tm}, \mathrm{EqTy}, \mathrm{EqTm}\}$ is the syntactic class of the non-abstracted boundary $b$.

The placeholder $\square$ in a boundary $\mathscr{B}$ may be filled with an argument $e$, called the head, to give a judgement $\mathcal{B} \in$, as follows:

$$
\begin{aligned}
(\square \text { type }) A & =(A \text { type }), \\
(\square: A) t & =(t: A), \\
(A \equiv B \text { by } \square) \star & =(A \equiv B \text { by } \star), \\
(s \equiv t: A \text { by } \square) \star & =(s \equiv t: A \text { by } \star), \\
(\{x: A\} \mathcal{B})\{x\} e & =(\{x: A\} \mathscr{B} e) .
\end{aligned}
$$

We also define the operation $\mathscr{B} e \equiv e^{\prime}$ which turns an object boundary $\mathscr{B}$ into an equation:

$$
\begin{aligned}
(\square \text { type }) A \equiv B & =(A \equiv B \text { by } \star), \\
(\square: A) s \equiv t & =(s \equiv t: A \text { by } \star), \\
(\{x: A\} B)\{x\} e \equiv\{x\} e^{\prime} & =\left(\{x: A\} B\left(e \equiv e^{\prime}\right) .\right.
\end{aligned}
$$

Example 2.2. If the symbols $A$ and Id have arities

$$
(\mathrm{Ty},[]), \quad \text { and }(\mathrm{Ty},[(\mathrm{Ty}, 0),(\mathrm{Tm}, 0),(\mathrm{Tm}, 0)]) \text {, }
$$

respectively, then the boundaries

$$
\{x: \mathrm{A}\}\{y: \mathrm{A}\} \square: \operatorname{Id}(\mathrm{A}, x, y) \quad \text { and } \quad\{x: \mathrm{A}\}\{y: \mathrm{A}\} x \equiv y: \mathrm{A} \text { by }
$$

may be filled with heads $\{x\}\{y\} x$ and $\{x\}\{y\} \star$ to yield abstracted judgements

$$
\{x: \mathrm{A}\}\{y: \mathrm{A}\} x: \operatorname{Id}(\mathrm{A}, x, y) \quad \text { and } \quad\{x: \mathrm{A}\}\{y: \mathrm{A}\} x \equiv y: \mathrm{A} \text { by } \star_{\mathrm{Tm}} .
$$


Information about available metavariables is collected in a metavariable context, which is a finite list $\Theta=\left[\mathrm{M}_{1}: \mathscr{B}_{1}, \ldots, \mathrm{M}_{n}: \mathscr{B}_{n}\right]$, also construed as a map, assigning to each metavariable $\mathrm{M}_{i}$ a boundary $\mathscr{B}_{i}$. In Section 2.6, $\Theta$ will provide typing of metavariable and premises of an inference rule, while the level of raw syntax it just determines metavariable arities. That is, $\Theta$ assigns the metavariable arity $\operatorname{ar}\left(\mathcal{B}_{i}\right)$ to $\mathrm{M}_{i}$.

A metavariable context $\Theta=\left[\mathrm{M}_{1}: \mathscr{B}_{1}, \ldots, \mathrm{M}_{n}: \mathscr{B}_{n}\right]$ may be restricted to a metavariable context $\Theta_{(i)}=\left[\mathrm{M}_{1}: \mathscr{B}_{1}, \ldots, \mathrm{M}_{i-1}: \mathscr{B}_{i-1}\right]$.

The metavariable context $\Theta$ is syntactically well formed when each $\mathscr{B}_{i}$ is a syntactically well-formed boundary over $\Sigma$ and $\Theta_{(i)}$. In addition each $\mathscr{B}_{i}$ must be closed, i.e., contain no free variables.

We also define the set of the object metavariables of $\Theta$ to be

$$
|\Theta|_{\text {obj }}=\left\{\mathrm{M}_{i} \mid \mathscr{B}_{i} \text { is an object boundary }\right\} \text {. }
$$

A variable context $\Gamma=\left[\mathrm{a}_{1}: A_{1}, \ldots, \mathrm{a}_{n}: A_{n}\right]$ over a metavariable context $\Theta$ is a finite list of pairs written as $\mathrm{a}_{i}: A_{i}$. It is considered syntactically valid when the variables $\mathrm{a}_{1}, \ldots, \mathrm{a}_{n}$ are all distinct, and for each $i$ the type expression $A_{i}$ is valid with respect to the signature and the metavariable arities assigned by $\Theta$, and the free variables occurring in $A_{i}$ are among $\mathrm{a}_{1}, \ldots, \mathrm{a}_{i-1}$. A variable context $\Gamma$ yields a finite map, also denoted $\Gamma$, defined by $\Gamma\left(\mathrm{a}_{i}\right)=A_{i}$. The domain of $\Gamma$ is the set $|\Gamma|=\left\{\mathrm{a}_{1}, \ldots, \mathrm{a}_{n}\right\}$.

A context is a pair $\Theta ; \Gamma$ consisting of a metavariable context $\Theta$ and a variable context $\Gamma$ over $\Theta$. A syntactic entity is considered syntactically valid over a signature and a context $\Theta ; \Gamma$ when all symbol and metavariable applications respect the assigned arities, the free variables are among $|\Gamma|$, and all bound variables are properly abstracted. It goes without saying that we always require all syntactic entities to be valid in this sense.

A (hypothetical) judgement has the form

$$
\Theta ; \Gamma \vdash \mathcal{G},
$$

where $\Theta ; \Gamma$ is a context and $\mathscr{I}$ is an abstracted judgement over $\Theta ; \Gamma$. In a hypothetical judgement

$$
\Theta ; \mathrm{a}_{1}: A_{1}, \ldots, \mathrm{a}_{n}: A_{n} \vdash\left\{x_{1}: B_{1}\right\} \cdots\left\{x_{m}: B_{m}\right\} j
$$

the hypotheses are split between the variable context $\mathrm{a}_{1}: A_{1}, \ldots, \mathrm{a}_{n}: A_{n}$ on the left of $\vdash$, and the abstraction $\left\{x_{1}: B_{1}\right\} \cdots\left\{x_{m}: B_{m}\right\}$ on the right. The former lists the global hypotheses that interact with other judgements, and the latter the hypotheses that are local to the judgement. In our experience such a separation is quite useful, because it explicitly marks the part of the context that is abstracted when a variable-binding symbol is applied to its arguments.

A (hypothetical) boundary is formed in the same fashion, as

$$
\Theta ; \Gamma \vdash \mathscr{B} \text {. }
$$

We read it as asserting that $\mathcal{B}$ is a well-typed boundary over the context $\Theta ; \Gamma$.

2.5. Instantiations. Let us spell out how how to instantiate metavariables with arguments. An instantiation of a metavariable context $\Xi=\left[\mathrm{M}_{1}: \mathscr{B}_{1}, \ldots, \mathrm{M}_{n}: \mathscr{B}_{n}\right]$ over a context $\Theta ; \Gamma$ is a list representing a map

$$
\left\langle\mathrm{M}_{1} \mapsto e_{1}, \ldots, \mathrm{M}_{n} \mapsto e_{n}\right\rangle,
$$

where $e_{i}$ 's are arguments over $\Theta ; \Gamma$ such that $\operatorname{ar}\left(\mathscr{B}_{i}\right)=\operatorname{ar}\left(e_{i}\right)$. We sometimes write $I \in$ $\operatorname{Inst}(\Xi, \Theta, \Gamma)$ when $I$ is such an instantiation. 
For $k \leq n$, define the restriction

$$
I_{(k)}=\left\langle\mathrm{M}_{1} \mapsto e_{1}, \ldots, \mathrm{M}_{k-1} \mapsto e_{k-1}\right\rangle .
$$

We sometimes write $I_{(\mathrm{M})}$ to indicate the initial segment up to the given metavariable $\mathrm{M} \in|I|$. We use the same notation for initial segments of sequences in general, e.g., if $\vec{x}=\left(x_{1}, \ldots, x_{n}\right)$ then $\vec{x}_{(k)}=\left(x_{1}, \ldots, x_{k-1}\right)$.

An instantiation $I$ acts on an expression $e$ to give an expression $I_{*} e$ by:

$$
\begin{gathered}
I_{*} \mathrm{a}=\mathrm{a}, \quad I_{*} x=x, \quad I_{*} \star=\star, \\
I_{*}(\{x\} e)=\{x\}\left(I_{*} e\right), \quad I_{*}(\mathrm{M}(\vec{t}))=e\left[I_{*} \vec{t} / \vec{x}\right] \text { if } I(\mathrm{M})=\{\vec{x}\} e, \\
I_{*}\left(\mathrm{~S}\left(\vec{e}^{\prime}\right)\right)=\mathrm{S}\left(I_{*} \vec{e}^{\prime}\right), \quad I_{*} \mathrm{M}(\vec{t})=\mathrm{M}\left(I_{*} \vec{t}\right) \text { if } \mathrm{M} \notin|I| .
\end{gathered}
$$

The action on abstracted judgements is given by

$$
\begin{aligned}
I_{*}(A \text { type }) & =\left(I_{*} A \text { type }\right), \\
I_{*}(t: A) & =\left(I_{*} t: I_{*} A\right), \\
I_{*}(A \equiv B \text { by } \star) & =\left(I_{*} A \equiv I_{*} B \text { by } \star\right), \\
I_{*}(s \equiv t: A \text { by } \star) & =\left(I_{*} A \equiv I_{*} B \text { by } \star\right), \\
I_{*}(\{x: A\} \mathcal{G}) & =\left(\left\{x: I_{*} A\right\} I_{*} \mathcal{G}\right) .
\end{aligned}
$$

An abstracted boundary may be instantiated analogously.

Given $I$ of $\Xi$ over $\Theta ; \Gamma$, and $\Delta=\left[x_{1}: A_{1}, \ldots, x_{n}: A_{n}\right]$ over $\Theta$ such that $|\Gamma| \cap|\Delta|=\emptyset$, we define $\Gamma, I_{*} \Delta$ to be the variable context

$$
\Gamma, x_{1}: I_{*} A_{1}, \ldots, x_{n}: I_{*} A_{n}
$$

Note that $I_{*} \Delta$ by itself is not a valid variable context. A judgement $\Xi ; \Delta \vdash \mathcal{G}$ may be instantiated to $\Theta ; \Gamma, I_{*} \Delta \vdash I_{*} \mathscr{Y}$. A hypothetical boundary can be instantiated analogously.

2.6. Raw rules. An inference rule in type theory is a template that generates a family of closure rules constituting a deductive system. In our setting, a raw rule is a hypothetical judgement of the form $\Theta ;[] \vdash j$, which we display as

$$
\Theta \Longrightarrow j
$$

It is an object rule when $j$ is an object judgement, and an equality rule when $j$ is an equality judgement. The designation "raw" signals that, even though a raw rule is syntactically sensible, it may be quite unreasonable from a type-theoretic point of view.

Given a rule $R=\left(\mathrm{M}_{1}: \mathcal{B}_{1}, \ldots, \mathrm{M}_{n}: \mathbb{B}_{n} \Longrightarrow 6[e)\right.$, along with an instantiation $I=$ $\left\langle\mathrm{M}_{1} \mapsto e_{1}, \ldots, \mathrm{M}_{n} \mapsto e_{n}\right\rangle$ of its premises over $\Theta ; \Gamma$, the rule instantiation $I_{*} R$ is the closure rule $\left(\left[p_{1}, \ldots, p_{n}, q\right], r\right)$ where $p_{i}$ is

$$
\Theta ; \Gamma \vdash\left(I_{(i) *} \mathcal{B}_{i}\right) e_{i},
$$

$q$ is $\Theta ; \Gamma \vdash I_{*} b$, and $r$ is $\Theta ; \Gamma \vdash I_{*}(b[e)$. In this way a raw rule generates a family of closure rules, indexed by instantiations. The premise $q$ is needed only in various meta-theoretic inductive arguments, as it ensures the well-formedness of the boundary of the conclusion. In practice, we use the "economic" variant $\left(\left[p_{1}, \ldots, p_{n}\right], r\right)$, which is easily seen to be admissible once Theorem 2.19 is established. 
Example 2.3. We may translate traditional ways of presenting rules to raw rules easily. For example, consider the formation rule for dependent products, which might be written as

$$
\frac{\Gamma \vdash A \text { type } \quad \Gamma, x: A \vdash B \text { type }}{\Gamma \vdash \Pi(A,\{x\} B) \text { type }}
$$

To be quite precise, the above is a family of closure rules, indexed by meta-level parameters $\Gamma, A$, and $B$ ranging over suitable syntactic entities. The template which generates such a family might be written as

$$
\frac{\vdash \mathrm{A} \text { type } \quad x: \mathrm{A} \vdash \mathrm{B}(x) \text { type }}{\vdash \Pi(\mathrm{A},\{x\} \mathrm{B}(x)) \text { type }}
$$

Indeed, there is no need to mention $\Gamma$ because it is always present, and we have replaced the parameters $A$ and $B$ with metavariables $\mathrm{A}$ and $\mathrm{B}$ (notice the change of fonts) to obtain bona-fide syntactic expressions. Next, observe that the premises amount to specifying an abstracted boundary for each metavariable, which brings us to

$$
\frac{\mathrm{A}:(\square \text { type }) \quad \mathrm{B}:(\{x: \mathrm{A}\} \square \text { type })}{\Pi(\mathrm{A},\{x\} \mathrm{B}(x)) \text { type }}
$$

By writing everything in a single line we obtain a raw rule

$$
\mathrm{A}:(\square \text { type }), \mathrm{B}:(\{x: \mathrm{A}\} \square \text { type }) \Longrightarrow \Pi(\mathrm{A},\{x\} \mathrm{B}(x)) \text { type. }
$$

The original family of closure rules is recovered when the above raw rule is instantiated with $\langle\mathrm{A} \mapsto A, \mathrm{~B} \mapsto\{x\} B\rangle$ where $A$ and $B$ are type expressions over (a metavariable context and) a variable context $\Gamma$.

We next define congruence and metavariable rules. These feature in every type theory.

Definition 2.4. The congruence rules associated with a raw object rule $R$

$$
\mathrm{M}_{1}: \mathscr{B}_{1}, \ldots, \mathrm{M}_{n}: \mathscr{B}_{n} \Longrightarrow b e
$$

are closure rules, with

$$
I=\left\langle\mathrm{M}_{1} \mapsto f_{1}, \ldots, \mathrm{M}_{n} \mapsto f_{n}\right\rangle \quad \text { and } \quad J=\left\langle\mathrm{M}_{1} \mapsto g_{1}, \ldots, \mathrm{M}_{n} \mapsto g_{n}\right\rangle,
$$

of the form

$$
\begin{array}{ll}
\Theta ; \Gamma \vdash\left(I_{(i) *} \mathcal{B}_{i}\right) f_{i} & \text { for } i=1, \ldots, n \\
\Theta ; \Gamma \vdash\left(J_{(i) *} \mathcal{B}_{i}\right) g_{i} & \text { for } i=1, \ldots, n \\
\Theta ; \Gamma \vdash\left(I_{(i) *} \mathcal{B}_{i}\right) f_{i} \equiv g_{i} & \text { for object boundary } \mathcal{B}_{i} \\
\frac{\Theta ; \Gamma \vdash I_{*} B \equiv J_{*} B}{-} & \text { if } \mathfrak{b}=(\square: B) \\
\Theta ; \Gamma \vdash\left(I_{*} b\right) & I_{*} e \equiv J_{*} e
\end{array}
$$

Metavariables have their own formation and congruence rules, akin to specific and congruence rules.

Definition 2.5. Given a context $\Theta ; \Gamma$ over $\Sigma$ with

$$
\Theta=\left[\mathrm{M}_{1}: \mathscr{B}_{1}, \ldots, \mathrm{M}_{n}: \mathcal{B}_{n}\right] \text { and } \mathscr{B}_{k}=\left(\left\{x_{1}: A_{1}\right\} \cdots\left\{x_{m}: A_{m}\right\} \mathfrak{b}\right),
$$


the metavariable rules for $\mathrm{M}_{k}$ are the closure rules of the form

$$
\begin{aligned}
& \Theta ; \Gamma \vdash t_{j}: A_{j}\left[\vec{t}_{(j)} / \vec{x}_{(j)}\right] \text { for } j=1, \ldots, m \\
& \frac{\Theta ; \Gamma \vdash b[\vec{t} / \vec{x}]}{\Theta ; \Gamma \vdash(b[\vec{t} / \vec{x}]) \mathrm{M}_{k}(\vec{t})}
\end{aligned}
$$

where $\vec{x}=\left(x_{1}, \ldots, x_{m}\right)$ and $\vec{t}=\left(t_{1}, \ldots, t_{m}\right)$. Furthermore, if $\boldsymbol{b}$ is an object boundary, then the metavariable congruence rules for $\mathrm{M}_{k}$ are the closure rules of the form

$$
\begin{array}{lr}
\Theta ; \Gamma \vdash s_{j}: A_{j}\left[\vec{s}_{(j)} / \vec{x}_{(j)}\right] & \text { for } j=1, \ldots, m \\
\Theta ; \Gamma \vdash t_{j}: A_{j}\left[\vec{t}_{(j)} / \vec{x}_{(j)}\right] & \text { for } j=1, \ldots, m \\
\Theta ; \Gamma \vdash s_{j} \equiv t_{j}: A_{j}\left[\vec{s}_{(j)} / \vec{x}_{(j)}\right] & \text { for } j=1, \ldots, m \\
\Theta ; \Gamma \vdash C[\vec{s} / \vec{x}] \equiv C[\vec{t} / \vec{x}] & \text { if } \mathcal{b}=(\square: C) \\
\hline \Theta ; \Gamma \vdash(b[\vec{s} / \vec{x}]) \mathrm{M}_{k}(\vec{s}) \equiv \mathrm{M}_{k}(\vec{t})
\end{array}
$$

where $\vec{s}=\left(s_{1}, \ldots, s_{m}\right)$ and $\vec{t}=\left(t_{1}, \ldots, t_{m}\right)$.

Once again, presuppositions may be elided safely from the above rules to yield the following admissible "economic" versions:

$$
\begin{aligned}
& \Theta ; \Gamma \vdash\left(I_{(i) *} \mathcal{B}_{i}\right)\left[f_{i} \quad \text { for equation boundary } \mathscr{B}_{i}\right. \\
& \frac{\left.\Theta ; \Gamma \vdash\left(I_{(i) *} \mathcal{B}_{i}\right)\right] f_{i} \equiv g_{i}}{\Theta ; \Gamma \vdash\left(I_{*} b\right)\left[I_{*} e \equiv J_{*} e\right.} \\
& \frac{\Theta ; \Gamma \vdash t_{j}: A_{j}\left[\vec{t}_{(j)} / \vec{x}_{(j)}\right] \quad \text { for } j=1, \ldots, m}{\Theta ; \Gamma \vdash(b[\vec{t} / \vec{x}]) \mathrm{M}_{k}(\vec{t})} \\
& \frac{\Theta ; \Gamma \vdash s_{j} \equiv t_{j}: A_{j}\left[\vec{s}_{(j)} / \vec{x}_{(j)}\right] \quad \text { for } j=1, \ldots, m}{\Theta ; \Gamma \vdash(b[\vec{s} / \vec{x}]) \mathrm{M}_{k}(\vec{s}) \equiv \mathrm{M}_{k}(\vec{t})}
\end{aligned}
$$

2.7. Type theories. A type theory in its basic form is a collection of rules that generate a deductive system. While this is too permissive a notion from a type-theoretic standpoint, it is nevertheless useful enough to deserve a name.

Definition 2.6. A raw type theory $T$ over a signature $\Sigma$ is a family of raw rules over $\Sigma$, called the specific rules of $T$. The associated deductive system of $T$ consists of:

(1) the structural rules over $\Sigma$ :

(a) the variable, metavariable, and abstraction rules (Definition 2.5 and Figure 2),

(b) the equality rules, (Figure 3),

(c) the boundary rules (Figure 4);

(2) the instantiations of the specific rules of $T$;

(3) for each specific object rule of $T$, the instantiations of the associated congruence rule (Definition 2.4). 


$$
\begin{aligned}
& \text { TT-MetA } \\
& \Theta\left(\mathrm{M}_{k}\right)=\left\{x_{1}: A_{1}\right\} \cdots\left\{x_{m}: A_{m}\right\} \mathcal{b} \\
& \text { TT-VAR } \\
& \Theta ; \Gamma \vdash t_{j}: A_{j}\left[\vec{t}_{(j)} / \vec{x}_{(j)}\right] \quad \text { for } j=1, \ldots, m \\
& \frac{\mathrm{a} \in|\Gamma|}{\Theta ; \Gamma \vdash \mathrm{a}: \Gamma(\mathrm{a})} \\
& \Theta ; \Gamma \vdash \mathfrak{b}[\vec{t} / \vec{x}] \\
& \Theta ; \Gamma \vdash(b[\vec{t} / \vec{x}]) \mathrm{M}_{k}(\vec{t}) \\
& \text { TT-Meta-Congr } \\
& \Theta\left(\mathrm{M}_{k}\right)=\left\{x_{1}: A_{1}\right\} \cdots\left\{x_{m}: A_{m}\right\} \mathfrak{b} \\
& \Theta ; \Gamma \vdash s_{j}: A_{j}\left[\vec{s}_{(j)} / \vec{x}_{(j)}\right] \quad \text { for } j=1, \ldots, m \\
& \Theta ; \Gamma \vdash t_{j}: A_{j}\left[\vec{t}_{(j)} / \vec{x}_{(j)}\right] \quad \text { for } j=1, \ldots, m \\
& \Theta ; \Gamma \vdash s_{j} \equiv t_{j}: A_{j}\left[\vec{s}_{(j)} / \vec{x}_{(j)}\right] \quad \text { for } j=1, \ldots, m \\
& \frac{\Theta ; \Gamma \vdash C[\vec{s} / \vec{x}] \equiv C[\vec{t} / \vec{x}] \quad \text { if } b=(\square: C)}{\Theta ; \Gamma \vdash(\mathcal{b}[\vec{s} / \vec{x}]) \mathrm{M}_{k}(\vec{s}) \equiv \mathrm{M}_{k}(\vec{t})} \\
& \text { TT-ABstR } \\
& \frac{\Theta ; \Gamma \vdash A \text { type } \quad \mathrm{a} \notin|\Gamma| \quad \Theta ; \Gamma, \mathrm{a}: A \vdash \mathscr{G}[\mathrm{a} / x]}{\Theta ; \Gamma \vdash\{x: A\} \mathscr{G}}
\end{aligned}
$$

Figure 2: Variable, metavariable and abstraction closure rules

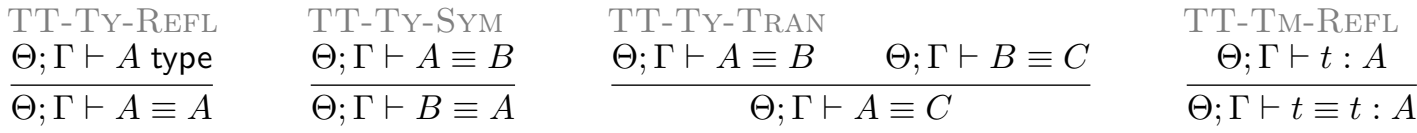

$$
\begin{aligned}
& \text { TT-TM-SYM TT-TM-Tran TT-Conv-TM } \\
& \frac{\Theta ; \Gamma \vdash s \equiv t: A}{\Theta ; \Gamma \vdash t \equiv s: A} \quad \frac{\Theta ; \Gamma \vdash s \equiv t: A \quad \Theta ; \Gamma \vdash t \equiv u: A}{\Theta ; \Gamma \vdash s \equiv u: A} \quad \frac{\Theta ; \Gamma \vdash t: A \quad \Theta ; \Gamma \vdash A \equiv B}{\Theta ; \Gamma \vdash t: B} \\
& \text { TT-CONV-EQ } \\
& \frac{\Theta ; \Gamma \vdash s \equiv t: A \quad \Theta ; \Gamma \vdash A \equiv B}{\Theta ; \Gamma \vdash s \equiv t: B}
\end{aligned}
$$

Figure 3: Equality closure rules

The rules of a raw type theory do not impose any conditions on the contexts, although they only ever extend variable contexts with well-formed types. When a well-formed context is needed, the auxiliary rules in Figure 5 are employed.

With the notion of raw type theory in hand, we may define concepts that employ derivability.

Definition 2.7. An instantiation $I=\left\langle M_{1} \mapsto e_{1}, \ldots, M_{n} \mapsto e_{n}\right\rangle$ of a metavariable context $\Xi=\left[M_{1}: \mathscr{B}_{1}, \ldots, M_{n}: \mathscr{B}_{n}\right]$ over $\Theta ; \Gamma$ is derivable when $\Theta ; \Gamma \vdash\left(I_{(k) *} \mathcal{B}_{k}\right) e_{k}$ for $k=1, \ldots, n$.

Definition 2.8. Instantiations

$$
I=\left\langle\mathrm{M}_{1} \mapsto e_{1}, \ldots, \mathrm{M}_{n} \mapsto e_{n}\right\rangle \quad \text { and } \quad J=\left\langle\mathrm{M}_{1} \mapsto f_{1}, \ldots, \mathrm{M}_{n} \mapsto f_{n}\right\rangle
$$




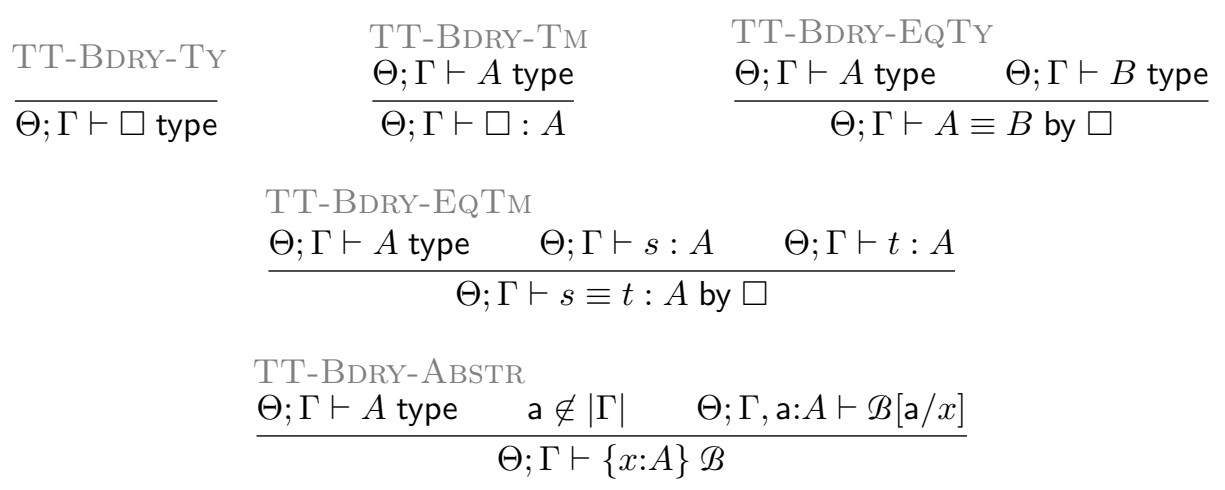

Figure 4: Well-formed abstracted boundaries

$\frac{\text { MCTX-EMPTy }}{\vdash[] \text { mctx }}$
VCTX-EMPTY
$\frac{\vdash \Theta \text { mctx }}{\Theta ; \Gamma \vdash[] \text { vctx }}$

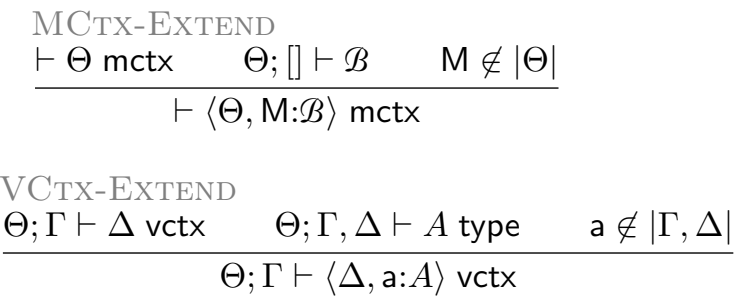

Figure 5: Well-formed contexts

over $\Theta ; \Gamma$ are judgementally equal when, for $k=1, \ldots, n$, if $\mathscr{B}_{k}$ is an object boundary then $\Theta ; \Gamma \vdash\left(I_{(k) *} \mathcal{B}_{k}\right) e_{k} \equiv f_{k}$.

Definition 2.9. A raw rule $\Xi \Longrightarrow j$ is derivable when it is derivable qua judgement. It is admissible when, for every derivable instantiation $I=\left\langle M_{1} \mapsto e_{1}, \ldots, M_{n} \mapsto e_{n}\right\rangle$ of $\Xi$ over $\Theta ; \Gamma$ we have $\Theta ; \Gamma \vdash I_{*} j$.

If $I$ is an instantiation of $\Xi=\left[\mathrm{M}_{1}: \mathscr{B}_{1}, \ldots, \mathrm{M}_{m}: \mathscr{B}_{m}\right]$ over $\Theta ; \Delta$, and $J$ is an instantiation of $\Theta$ over $\Psi ; \Gamma$ such that $|\Gamma| \cap|\Delta|=\emptyset$, their composition $J \circ I$ is the instantiation of $\Xi$ over $\Psi ; \Gamma, J_{*} \Delta$ defined by

$$
(J \circ I)(\mathrm{M})=J_{*}(I(\mathrm{M})) .
$$

Composition of instantiations is associative. It also preserves derivability, which can be proved easily once Theorem 2.17 is established.

It will be useful to know that derivability only needs to be checked for instantiations over the empty variable context. For this purpose, define the promotion of

$$
\Theta ; \Gamma \vdash \mathcal{G}
$$

to be the judgement

$$
(\Theta, \Gamma) ;[] \vdash \mathcal{G},
$$

in which the free variables are promoted to metavariables. (We could obfuscate what we just said by being more precise: if $\Gamma=\left[\mathrm{a}_{1}: A_{1}, \ldots, \mathrm{a}_{n}: A_{n}\right]$, the promotion is the judgement

$$
\left(\Theta, \mathrm{a}_{1}^{\prime}: A_{1}^{\prime}, \ldots, \mathrm{a}_{n}^{\prime}: A_{n}^{\prime}\right) ;[] \vdash \mathscr{I}\left[\overrightarrow{\mathrm{a}}^{\prime} / \overrightarrow{\mathrm{a}}\right]
$$


in which $\mathrm{a}_{1}^{\prime}, \ldots, \mathrm{a}_{n}^{\prime}$ are fresh and $\left.A_{i}^{\prime}=A_{i}\left[\overrightarrow{\mathrm{a}}_{(i)}{ }_{(i)} / \overrightarrow{\mathrm{a}}_{(i)}\right].\right)$ Note that $\vdash(\Theta, \Gamma)$ mctx is derivable if, and only if, both $\vdash \Theta$ mctx and $\Theta \vdash \Gamma$ vctx are derivable.

Proposition 2.10. A raw type theory derives $\Theta ; \Gamma \vdash \mathcal{G}$ if, and only if, it derives the promotion $(\Theta, \Gamma) ;[] \vdash \mathcal{G}$.

Proof. To pass between the original variable context and its promotion, swap applications of TT-VAR with corresponding applications of TT-META.

Raw rules do not impose any well-typedness conditions on the premises and the conclusion. We may rectify this by requiring that their boundaries be derivable, as follows.

Definition 2.11. A raw rule $\Theta \Longrightarrow b \boxminus$ is a finitary rule with respect to a raw type theory $T$ when $\vdash \Theta$ mctx and $\Theta$; [] $\vdash \mathfrak{b}$ are derivable. A finitary type theory is a raw type theory $T$ whose rules are finitary with respect to $T$.

According to the above definition, the justification that a rule is finitary may rely on the rule itself. If so desired, such circularity may be proscribed by imposition of a well-found order on the rules, with the requirement that the finitary character of each rule be established using only the rules preceding it, see [BHL20] for further details.

Example 2.12. A finitary type theory is well behaved in many respects, but may still be "non-standard". Assuming N, O and S are respectively a type constant, a term constant, and a unary term symbol, the rules

$$
[] \Longrightarrow \mathrm{N} \text { type, } \quad[] \Longrightarrow \mathrm{O}: \mathrm{N}, \quad \mathrm{n}:(\square: \mathrm{N}) \Longrightarrow \mathrm{S}(\mathrm{S}(\mathrm{n})): \mathrm{N}
$$

constitute a finitary type theory. However, the third rule is troublesome because it posits a compound term $\mathrm{S}(\mathrm{S}(\mathrm{n}))$.

We avoid such anomalies by requiring that object rules only ever introduce generically applied symbols. For this purpose, define a rule-boundary to be a hypothetical boundary of the form $\Theta ;[] \vdash \mathfrak{b}$, notated as $\Theta \Longrightarrow \mathfrak{b}$. The elements of $\Theta$ are the premises and $b$ is the conclusion boundary. We say that the rule-boundary is an object rule-boundary when $b$ is a type or a term boundary, and an equality rule-boundary when $b$ is an equality boundary. Next, given an object rule-boundary

$$
\mathrm{M}_{1}: \mathscr{B}_{1}, \ldots, \mathrm{M}_{n}: \mathscr{B}_{n} \Longrightarrow \mathcal{b} .
$$

its associated symbol arity is $\left(c,\left[\operatorname{ar}\left(\mathscr{B}_{1}\right), \ldots, \operatorname{ar}\left(\mathscr{B}_{n}\right)\right]\right)$, where $c \in\{\mathrm{Ty}, \mathrm{Tm}\}$ is the syntactic class of $\boldsymbol{b}$. Given a fresh symbol S, we assign it the associated arity and define the associated symbol rule to be

$$
\mathrm{M}_{1}: \mathscr{B}_{1}, \ldots, \mathrm{M}_{n}: \mathscr{B}_{n} \Longrightarrow b\left[\mathrm{~S}\left(\widehat{\mathrm{M}}_{i}, \ldots, \widehat{\mathrm{M}}_{n}\right)\right]
$$

where $\widehat{\mathrm{M}}_{i}$ is the generic application of the metavariable $\mathrm{M}_{i}$, defined as, assuming $\operatorname{ar}\left(\mathscr{B}_{i}\right)=$ $\left(c_{i}, n_{i}\right)$ :

(1) $\widehat{M}_{i}=\left\{x_{1}, \ldots, x_{n_{i}}\right\} \mathrm{M}_{i}\left(x_{1}, \ldots, x_{n_{i}}\right)$ when $c_{i} \in\{\mathrm{Ty}, \mathrm{Tm}\}$,

(2) $\widehat{M}_{i}=\left\{x_{1}, \ldots, x_{n_{i}}\right\} \star$ when $c_{i} \in\{\mathrm{EqTy}, \mathrm{EqTm}\}$.

Here then is our final notion of type theory.

Definition 2.13. A finitary type theory is standard if its specific object rules are symbol rules, and each symbol has precisely one associated rule. 
2.8. A review of meta-theorems. We recall from [HB21] meta-theorems that establish desirable structural properties of type theories. In the next section we prove several additional meta-theorems that we rely on subsequently.

First we have meta-theorems about raw type theories that are concerned with syntactic manipulations.

Proposition 2.14 (Renaming). If a raw type theory derives a judgement or a boundary, then it also derives its renaming.

Proposition 2.15 (Weakening). For a raw type theory:

(1) If $\Theta ; \Gamma_{1}, \Gamma_{2} \vdash \mathcal{G}$ then $\Theta ; \Gamma_{1}, \mathrm{a}: A, \Gamma_{2} \vdash \mathcal{G}$.

(2) If $\Theta_{1}, \Theta_{2} ; \Gamma \vdash \mathcal{G}$ then $\Theta_{1}, \mathrm{M}: \mathcal{B}, \Theta_{2} ; \Gamma \vdash \mathcal{G}$.

An analogous statement holds for boundaries.

It is understood that in the above statements, and the subsequent ones, we tacitly assume whatever syntactic conditions are needed to ensure that all entities are well-formed. For example, in Proposition 2.15 we require a $\notin\left|\Gamma_{1}, \Gamma_{2}\right|$ and that $A$ be a syntactically valid type expression for $\Theta ; \Gamma_{1}$.

Theorem 2.16 (Admissibility of substitution). In a raw type theory the substitution rules from Figure 6 are admissible.

$$
\begin{array}{lrl}
\begin{array}{l}
\text { TT-Subst } \\
\Theta ; \Gamma \vdash\{x: A\} \mathscr{I} \quad \Theta ; \Gamma \vdash t: A
\end{array} & \frac{\Theta ; \Gamma \vdash\{x: A\} \mathscr{B} \quad \Theta ; \Gamma \vdash t: A}{\Theta ; \Gamma \vdash \mathscr{G}[t / x]}
\end{array}
$$

$$
\begin{aligned}
& \text { TT-Subst-EQTy } \\
& \frac{\Theta ; \Gamma \vdash\{x: A\}\{\vec{y}: \vec{B}\} C \text { type } \quad \Theta ; \Gamma \vdash s: A \quad \Theta ; \Gamma \vdash t: A \quad \Theta ; \Gamma \vdash s \equiv t: A}{\Theta ; \Gamma \vdash\{\vec{y}: \vec{B}[s / x]\} C[s / x] \equiv C[t / x]} \\
& \text { TT-SuBst-EQTM } \\
& \frac{\Theta ; \Gamma \vdash\{x: A\}\{\vec{y}: \vec{B}\} u: C \quad \Theta ; \Gamma \vdash s: A \quad \Theta ; \Gamma \vdash t: A \quad \Theta ; \Gamma \vdash s \equiv t: A}{\Theta ; \Gamma \vdash\{\vec{y}: \vec{B}[s / x]\} u[s / x] \equiv u[t / x]: C[s / x]} \\
& \text { TT-Conv-ABstr } \\
& \frac{\Theta ; \Gamma \vdash\{x: A\} \mathscr{I} \quad \Theta ; \Gamma \vdash B \text { type } \quad \Theta ; \Gamma \vdash A \equiv B}{\Theta ; \Gamma \vdash\{x: B\} \mathscr{I}}
\end{aligned}
$$

Figure 6: Admissible substitution rules

Next we have admissibility of instantiations.

Theorem 2.17 (Admissibility of instantiations). In a raw type theory, let I be a derivable instantiation of $\Xi$ over $\Theta ; \Delta$. If $\Xi ; \Gamma \vdash \mathcal{G}$ is derivable and $|\Delta| \cap|\Gamma|=\emptyset$ then $\Theta ; \Delta, I_{*} \Gamma \vdash I_{*} \mathcal{I}$ is derivable, and similarly for boundaries.

Theorem 2.18. In a raw type theory, let $I$ and $J$ be judgementally equal derivable instantiations of $\Xi$ over $\Theta ; \Gamma$. Suppose $\Xi \vdash \Delta$ vctx and $|\Gamma| \cap|\Delta|=\emptyset$. If $\Xi ; \Delta \vdash \mathscr{B} \boxminus$ is a derivable object judgement then $\Theta ; \Gamma, I_{*} \Delta \vdash\left(I_{*} \mathscr{B}\right) I_{*} e \equiv J_{*} e$ is derivable. 
To obtain meta-theorems with genuine type-theoretic contents, we need to restrict to finitary type theories.

Theorem 2.19 (Presuppositivity). If a finitary type theory derives $\Theta \vdash \Gamma$ vctx, and $\Theta ; \Gamma \vdash$ $\mathscr{B} e$ then it derives $\Theta ; \Gamma \vdash \mathcal{B}$.

The next two theorems apply to standard type theories. The first one provides an inversion principle, and the second one guarantees that a term has at most one type, up to judgemental equality. Both rely on a candidate type that may be read off directly from the term.

Definition 2.20. Let $T$ be a standard type theory. The natural type $\tau_{\Theta ; \Gamma}(t)$ of a term expression $t$ with respect to a context $\Theta ; \Gamma$ is defined by:

$$
\begin{aligned}
\tau_{\Theta ; \Gamma}(\mathrm{a}) & =\Gamma(a), \\
\tau_{\Theta ; \Gamma}\left(\mathrm{M}\left(t_{1}, \ldots, t_{m}\right)\right)=A\left[t_{1} / x_{1}, \ldots, t_{m} / x_{m}\right] & \\
& \text { where } \Theta(\mathrm{M})=\left(\left\{x_{1}: A_{1}\right\} \cdots\left\{x_{m}: A_{m}\right\} \square: A\right) \\
\tau_{\Theta ; \Gamma}\left(\mathrm{S}\left(e_{1}, \ldots, e_{n}\right)\right)=\left\langle\mathrm{M}_{1} \mapsto e_{1}, \ldots, \mathrm{M}_{n} \mapsto e_{n}\right\rangle_{*} B & \\
& \text { where the symbol rule for } \mathrm{S} \text { is }
\end{aligned}
$$$$
\mathrm{M}_{1}: \mathscr{B}_{1}, \ldots, \mathrm{M}_{n}: B_{n} \Longrightarrow \mathrm{S}\left(\widehat{\mathrm{M}}_{1}, \ldots, \widehat{\mathrm{M}}_{n}\right): B \text {. }
$$

The following theorem is an inversion principle that recovers the "stump" of a derivation of a derivable object judgement.

Theorem 2.21 (Inversion theorem). If a standard finitary type theory derives a term judgement then it does so by a derivation which concludes with precisely one of the following rules:

(1) the variable rule TT-VAR,

(2) the metavariable rule TT-META,

(3) an instantiation of a symbol rule,

(4) the abstraction rule TT-ABSTR,

(5) the term conversion rule TT-Conv-TM of the form

$$
\frac{\Theta ; \Gamma \vdash t: \tau_{\Theta ; \Gamma}(t) \quad \Theta ; \Gamma \vdash \tau_{\Theta ; \Gamma}(t) \equiv A}{\Theta ; \Gamma \vdash t: A}
$$

where $\tau_{\Theta ; \Gamma}(t) \neq A$.

Finally, in a standard type theory a term has at most one type, up to judgemental equality.

Theorem 2.22 (Uniqueness of typing). For a standard finitary type theory:

(1) If $\Theta ; \Gamma \vdash t: A$ and $\Theta ; \Gamma \vdash t: B$ then $\Theta ; \Gamma \vdash A \equiv B$.

(2) If $\Theta$; [] $\vdash \Gamma \operatorname{vctx}$ and $\Theta ; \Gamma \vdash s \equiv t: A$ and $\Theta ; \Gamma \vdash s \equiv t: B$ then $\Theta ; \Gamma \vdash A \equiv B$.

2.9. More meta-theorems. We state and prove several further meta-theorems.

Proposition 2.23. Let $T$ be a standard type theory and $I$ an instantiation of $\Xi$ over $\Theta ; \Gamma$. For a term expression $\mathrm{S}(\vec{e})$ it holds that $I_{*}\left(\tau_{\Xi ; \Delta}(\mathrm{S}(\vec{e}))\right)=\tau_{\Theta ; \Gamma, I_{*} \Delta}\left(I_{*} \mathrm{~S}(\vec{e})\right)$. 
Proof. Let $\mathrm{M}_{1}: \mathscr{B}_{1}, \ldots, \mathrm{M}_{n}: \mathscr{B}_{n} \Longrightarrow \mathrm{S}\left(\widehat{\mathrm{M}}_{1}, \ldots, \widehat{\mathrm{M}}_{n}\right): B$ be the symbol rule for $\mathrm{S}$. By unfolding the definition of the natural type we have

$$
\begin{aligned}
I_{*}\left(\tau_{\Xi ; \Delta}(\mathrm{S}(\vec{e}))\right) & =I_{*}\left(\left\langle\mathrm{M}_{1} \mapsto e_{1}, \ldots, \mathrm{M}_{n} \mapsto e_{n}\right\rangle_{*} B\right)=\left\langle\mathrm{M}_{1} \mapsto I_{*} e_{1}, \ldots, \mathrm{M}_{n} \mapsto I_{*} e_{n}\right\rangle_{*} B \\
& =\tau_{\Theta ; \Gamma, I_{*} \Delta}\left(\mathrm{S}\left(I_{*} \vec{e}\right)\right)=\tau_{\Theta ; \Gamma, I_{*} \Delta}\left(I_{*}(\mathrm{~S}(\vec{e}))\right)
\end{aligned}
$$

Note that $I$ acts purely syntactically and needs not be derivable for the equation to hold. It is also worth pointing out that the equation does not hold for metavariable term expressions. We now explicate two common usages of Theorem 2.21.

Corollary 2.24. In a standard type theory, suppose the rule for $\mathrm{S}$ is

$$
\mathrm{M}_{1}: \mathscr{B}_{1}, \ldots, \mathrm{M}_{n}: \mathscr{B}_{n} \Longrightarrow \mathcal{b}^{\prime} \mathrm{S}\left(\widehat{\mathrm{M}}_{1}, \ldots, \widehat{\mathrm{M}}_{n}\right) \text {. }
$$

If the theory derives $\Theta ; \Gamma \vdash b\left[\mathrm{~S}(\vec{e})\right.$ then it also derives $\Theta ; \Gamma\left(I_{(i) *} \mathcal{B}_{i}\right) e_{i}$ for all $i=1, \ldots, n$, where $I=\left\langle\mathrm{M}_{1} \mapsto e_{1}, \ldots, \mathrm{M}_{n} \mapsto e_{n}\right\rangle$.

Proof. By Theorem 2.21 the judgement is derived by an application of the symbol rule for $\mathrm{S}$, possibly followed by a conversion, whose premises are precisely the judgements of interest.

Corollary 2.25. If a standard type theory derives $\Theta ; \Gamma \vdash t$ : A then it also derives $\Theta ; \Gamma \vdash t$ : $\tau_{\Theta ; \Gamma}(t)$.

Proof. By Theorem 2.21, either $A=\tau_{\Theta, \Gamma}(t)$ and there is nothing to prove, or the derivations ends with

$$
\frac{\Theta ; \Gamma \vdash t: \tau_{\Theta ; \Gamma}(t) \quad \Theta ; \Gamma \vdash \tau_{\Theta ; \Gamma}(t) \equiv A}{\Theta ; \Gamma \vdash t: A}
$$

which contains the desired equality as a subderivation.

We next prove a statement about instantiations that needs a couple of preparatory lemmas.

Lemma 2.26. If a finitary type theory derives $\Theta \vdash \Gamma \operatorname{vctx}$ and $\Theta ; \Gamma \vdash \mathcal{B} e \equiv e^{\prime}$ then it derives $\Theta ; \Gamma \vdash \mathcal{B} e^{\prime}$.

Proof. We proceed by induction on the number of abstractions in the object boundary $\mathcal{B}$.

Case $\mathscr{B}=(\square$ type $), e=A$ and $e^{\prime}=B$ : Theorem 2.19 applied to the assumption $\Theta ; \Gamma \vdash A \equiv$ $B$ by $\star$ gives $\Theta ; \Gamma \vdash A \equiv B$ by $\square$, from which $\Theta ; \Gamma \vdash B$ type follows by inversion.

Case $\mathcal{B}=(\square: A)$ : Similar to the previous case.

Case $\mathcal{B}=\left(\{x: A\} \mathcal{B}^{\prime}\right)$ : Inversion on the assumption $\Theta ; \Gamma \vdash\{x: A\} \mathscr{B}^{\prime} e \equiv e^{\prime}$ gives

$$
\Theta ; \Gamma \vdash A \text { type } \quad \text { and } \quad \Theta ; \Gamma, \mathrm{a}: A \vdash\left(\mathscr{B}^{\prime}[\mathrm{a} / x]\right) e[\mathrm{a} / x] \equiv e^{\prime}[\mathrm{a} / x] \text {. }
$$

By induction hypothesis, the second judgement entails

$$
\Theta ; \Gamma, \mathrm{a}: A \vdash\left(\mathcal{B}^{\prime}[\mathrm{a} / x]\right) e^{\prime}[\mathrm{a} / x],
$$

which we may abstract to the desired form.

Lemma 2.27. In a finitary type theory, consider judgementally equal derivable instantiations $I$ and $J$ of $\Xi$ over $\Theta ; \Gamma$, and suppose $\Xi \vdash \Delta$ vatx and $\Xi ; \Delta \vdash \mathscr{B}$ such that $|\Delta| \cap|\Gamma|=\emptyset$. If $\Theta ; \Gamma, I_{*} \Delta \vdash\left(I_{*} \mathcal{B}\right) e$ is derivable then so is $\Theta ; \Gamma, I_{*} \Delta \vdash\left(J_{*} \mathcal{B}\right)$ e]. 
Proof. We proceed by structural induction on the derivation of $\Xi ; \Delta \vdash \mathscr{B}$.

Case TT-BDRY-TY: Trivial because $I_{*} \mathscr{B}=(\square$ type $)=J_{*} \mathscr{B}$.

Case TT-BDRY-TM: If the derivation ends with

$$
\frac{\Xi ; \Delta \vdash A \text { type }}{\Xi ; \Delta \vdash \square: A}
$$

then $\Theta ; \Gamma, I_{*} \Delta \vdash I_{*} A \equiv J_{*} A$ by Theorem 2.18 applied to the premise, hence we may convert $\Theta ; \Gamma, I_{*} \Delta \vdash e: I_{*} A$ to $\Theta ; \Gamma, I_{*} \Delta \vdash e: J_{*} A$.

Case TT-BDRY-EQTY: If the derivation ends with

$$
\frac{\Xi ; \Delta \vdash A \text { type } \quad \Xi ; \Delta \vdash B \text { type }}{\Xi ; \Delta \vdash A \equiv B \text { by } \square}
$$

then Theorem 2.18 applied to the premises gives us

$$
\Theta ; \Gamma, I_{*} \Delta \vdash I_{*} A \equiv J_{*} A \quad \text { and } \quad \Theta ; \Gamma, I_{*} \Delta \vdash I_{*} B \equiv J_{*} B .
$$

Together with the assumption $\Theta ; \Gamma, I_{*} \Delta \vdash I_{*} A \equiv I_{*} B$, these suffice to derive $\Theta ; \Gamma, I_{*} \Delta \vdash$ $J_{*} A \equiv J_{*} B$.

Case TT-BDry-EqTM: similar to TT-BDry-EQTy.

Case TT-BDry-ABstr: Suppose $e=\{x\} e^{\prime}$ and the derivation ends with

$$
\frac{\Xi ; \Delta \vdash A \text { type } \quad \mathrm{a} \notin|\Delta| \quad \Xi ; \Delta, \mathrm{a}: A \vdash \mathscr{B}^{\prime}[\mathrm{a} / x]}{\Xi ; \Delta \vdash\{x: A\} \mathscr{B}^{\prime}}
$$

where we may assume a $\notin|\Gamma|$ without loss of generality. Theorem 2.18 applied to the first premise derives

$$
\Theta ; \Gamma, I_{*} \Delta \vdash I_{*} A \equiv J_{*} A .
$$

By inverting the assumption $\Theta ; \Gamma, I_{*} \Delta \vdash\left\{x: I_{*} A\right\}\left(I_{*} \mathscr{B}^{\prime}\right) e$, and possibly renaming a free variable to a, we obtain

$$
\left.\Theta ; \Gamma, I_{*} \Delta \vdash I_{*} A \text { type and } \Theta ; \Gamma, I_{*} \Delta, \mathrm{a}: I_{*} A \vdash\left(\left(I_{*} \mathcal{B}^{\prime}\right) \Theta\right]\right)[\mathrm{a} / x] .
$$

Then the induction hypothesis for the second premise yields

$$
\left.\Theta ; \Gamma, I_{*} \Delta, \mathrm{a}: I_{*} A \vdash\left(\left(J_{*} \mathcal{B}^{\prime}\right) e\right]\right)[\mathrm{a} / x],
$$

which we may abstract to $\Theta ; \Gamma, I_{*} \Delta \vdash\left\{x: I_{*} A\right\}\left(J_{*} \mathcal{B}^{\prime}\right) e$ and apply TT-Conv-ABstr to convert it to the desired judgement $\Theta ; \Gamma, I_{*} \Delta \vdash\left\{x: J_{*} A\right\}\left(J_{*} \mathcal{B}^{\prime}\right) e$. The premise $\Theta ; \Gamma, I_{*} \Delta \vdash$ $J_{*} A$ type is derived by Theorem 2.19 from (2.1).

Proposition 2.28. In a finitary type theory, consider instantiations $I$ and $J$ of $\Xi$ over $\Theta ; \Gamma$, such that $\vdash \Xi$ mctx and $\Theta \vdash \Gamma$ vctx. If $I$ is derivable and $I$ and $J$ are judgementally equal then $J$ is derivable.

Proof. We prove the claim by induction on the length of $\Xi$. The base case is trivial. For the induction step we assume the statement, and show that is still holds when we extend $\Xi, I$ and $J$ by one more entry. Specifically, assume that

$$
\Xi ;[] \vdash \mathscr{B}, \quad \text { and } \quad \Theta ; \Gamma \vdash\left(I_{*} \mathscr{B}\right) e \text {, }
$$

and if $\mathcal{B}$ is an object boundary also that

$$
\Theta ; \Gamma \vdash\left(I_{*} \mathscr{B}\right) e \equiv e^{\prime} .
$$


Then we must demonstrate $\Theta ; \Gamma \vdash\left(J_{*} \mathcal{B}\right) e^{\prime}$.

If $\mathcal{B}$ is an equality boundary then applying Lemma 2.27 to $(2.2)$ gives $\Theta ; \Gamma \vdash\left(J_{*} \mathscr{B}\right) e$, and we are done because $e$ and $e^{\prime}$ are the same.

If $\mathcal{B}$ is an object boundary then applying Lemma 2.27 to $\Xi ;[] \vdash \mathscr{B}$ and (2.3) gives $\Theta ; \Gamma \vdash\left(J_{*} \mathcal{B}\right) e \equiv e^{\prime}$. The derivability of $\Theta ; \Gamma \vdash\left(J_{*} \mathscr{B}\right) e^{\prime}$ now follows from Lemma 2.26.

\section{The EqUALity CHECKING ALGORITHM}

The equality checking algorithm applies inference rules by pattern matching them against (parts of) the target equation. We therefore begin by studying the type-theoretic and syntactic properties of rules by which the soundness of pattern matching can be ensured.

3.1. Patterns and object-invertible rules. In order to derive $\Theta ; \Gamma \vdash j^{\prime}$ with the rule $\Xi \Longrightarrow j$, we must find an instantiation $I$ of $\Xi$ over $\Theta ; \Gamma$ such that $I_{*} j=j^{\prime}$. We shall be primarily interested in rules where such $I$ is unique, when it exists.

Definition 3.1. A raw rule $\Xi \Longrightarrow j$ is deterministic when for every judgement $\Theta ; \Gamma \vdash j^{\prime}$ there exists at most one instantiation $I$ of $\Xi$ over $\Theta ; \Gamma$ such that $I_{*} j=j^{\prime}$, called a matching instantiation.

We refrain from trying to characterize the deterministic rules, and instead observe that, given a deterministic rule

$$
R=\left(\mathrm{M}_{1}: \mathscr{B}_{1}, \ldots, \mathrm{M}_{n}: \mathscr{B}_{n} \Longrightarrow j\right)
$$

and a judgement $\Theta ; \Gamma \vdash j^{\prime}$ we may algorithmically compute $I$ such that $I_{*} j=j^{\prime}$, or decide that it does not exist. First of all, every object metavariable of $R$ must appear in $j$, or else $R$ would match in multiple ways the judgement $\Theta, \Theta^{\prime} ;[] \vdash j$, where $\Theta^{\prime}$ is a copy of $\Theta$ in which each $\mathrm{M}_{i}$ is replaced with $\mathrm{M}_{i}^{\prime}$. Therefore, for any instantiation

$$
I=\left\langle\mathrm{M}_{1} \mapsto e_{1}, \ldots, \mathrm{M}_{n} \mapsto e_{n}\right\rangle
$$

where $\operatorname{ar}\left(e_{i}\right)=\operatorname{ar}\left(\mathscr{B}_{i}\right)=\left(c_{i}, n_{i}\right)$ and $e_{i}=\left\{x_{1}, \ldots, x_{n_{i}}\right\} e_{i}^{\prime}$, the size of $I_{*} j$ equals or exceeds the size of each $e_{i}^{\prime}$. We may therefore look for an instantiation that matches $\Theta ; \Gamma \vdash j$ by exhaustively searching through all $e_{i}^{\prime}$ 's over $\Theta ; \Gamma$ whose sizes are bounded by the size of $j$, of which there are only finitely many. Of course, we are not suggesting that anyone should use such an exhaustive search in practice. Instead, we provide a simple syntactic criterion that makes a rule deterministic and easy to match against.

Definition 3.2. Patterns are expressions in which every metavariable occurs at most once either in an application without arguments $M()$, or in an argument of the form $\{\vec{x}\} \mathrm{M}(\vec{x})$, where $\vec{x}$ are the only bound variables in scope. They are described by the grammar in Figure 7.

$$
\begin{aligned}
\text { Type pattern } P::=\mathrm{M}() \mid \mathrm{S}\left(q_{1}, \ldots, q_{n}\right) & \text { if } \operatorname{mv}\left(q_{i}\right) \cap \operatorname{mv}\left(q_{j}\right)=\emptyset \text { for } i \neq j \\
\text { Term pattern } p::=\mathrm{S}\left(q_{1}, \ldots, q_{n}\right) & \text { if } \operatorname{mv}\left(q_{i}\right) \cap \operatorname{mv}\left(q_{j}\right)=\emptyset \text { for } i \neq j \\
\text { Argument pattern } q::=\{\vec{x}\} \mathrm{M}(\vec{x})|P| p &
\end{aligned}
$$

Figure 7: The syntax of patterns. 
Note that $\mathrm{M}()$ can only appear as a type pattern, but not as a term pattern. The reason for this lies in the definitions of computation rules (Definitions 3.18 and 3.19) which we shall see later on.

As defined, the patterns are linear in the sense that a metavariable cannot appear several times, and first-order because patterns may not appear under abstractions. Non-linearity is not an essential limitation, as we shall see shortly. The restriction to first-order patterns arises because in general a standard type theory may not satisfy the strengthening principle which states that if $\vdash\{x: A\} \mathscr{I}$ is derivable and $x \notin \mathrm{bv}(\mathscr{G})$ then $\vdash \mathscr{I}$ is derivable. The principle allows a higher-order pattern to safely extract an expression from within an abstraction, so long as no bound variables escape their scopes.

Example 3.3. The head of the conclusion of a symbol rule

$$
\mathrm{M}_{1}: \mathscr{B}_{1}, \ldots, \mathrm{M}_{n}: \mathcal{B}_{n} \Longrightarrow b \mathrm{~S}\left(\widehat{M}_{1}, \ldots, \widehat{M}_{n}\right)
$$

is a pattern because $\widehat{\mathrm{M}}_{i}$ has required form $\{x\} \mathrm{M}_{i}(\vec{x})$.

Example 3.4. Consider the $\beta$-rule for the first projection from a binary product:

$$
\frac{\vdash \text { A type } \quad \vdash \text { B type } \vdash \mathrm{s}: \mathrm{A} \quad \vdash \mathrm{t}: \mathrm{B}}{\vdash \text { fst }(\mathrm{A}, \mathrm{B}, \operatorname{pair}(\mathrm{A}, \mathrm{B}, \mathrm{s}, \mathrm{t})) \equiv \mathrm{s}: \mathrm{A}}
$$

The left-hand side of the conclusion is not a pattern because the metavariables $A$ and $B$ occur twice each. We may linearize the pattern at the cost of equational premises:

$$
\begin{aligned}
& \vdash \mathrm{A}_{1} \text { type } \quad \vdash \mathrm{A}_{2} \text { type } \quad \vdash \mathrm{B}_{1} \text { type } \vdash \mathrm{B}_{2} \text { type } \\
& \frac{\vdash \mathrm{s}: \mathrm{A}_{2} \quad \vdash \mathrm{t}: \mathrm{B}_{2} \quad \vdash \mathrm{A}_{1} \equiv \mathrm{A}_{2} \quad \vdash \mathrm{B}_{1} \equiv \mathrm{B}_{2}}{\vdash \mathrm{fst}\left(\mathrm{A}_{1}, \mathrm{~B}_{1}, \operatorname{pair}\left(\mathrm{A}_{2}, \mathrm{~B}_{2}, \mathrm{~s}, \mathrm{t}\right)\right) \equiv \mathrm{s}: \mathrm{A}_{1}}
\end{aligned}
$$

The new rule is inter-derivable with the original one. It is clear that the technique works generally and that it can be automated.

Example 3.5. Consider the rule stating that the identity function is the neutral element for composition:

$$
\frac{\vdash \text { A type } \quad \vdash \text { B type } \quad \vdash \mathrm{f}: \mathrm{A} \rightarrow \mathrm{B}}{\vdash \operatorname{compose}(\mathrm{A}, \mathrm{B}, \mathrm{f}, \lambda(\mathrm{A}, \mathrm{A},\{x\} x)) \equiv \mathrm{f}: \mathrm{A} \rightarrow \mathrm{B}}
$$

The left-hand side of the conclusion is not a pattern because $\lambda(A, A,\{x\} x)$ is not a pattern. Once again we can remedy the situation by introducing an additional equational premise:

$$
\begin{array}{cc}
\vdash \mathrm{A} \text { type } & \vdash \mathrm{B} \text { type } \quad \vdash \mathrm{f}: \mathrm{A} \rightarrow \mathrm{B} \\
\vdash \mathrm{i}: \mathrm{A} \rightarrow \mathrm{A} & \vdash \mathrm{i} \equiv \lambda(\mathrm{A}, \mathrm{A},\{x\} x): \mathrm{A} \rightarrow \mathrm{A} \\
& \vdash \operatorname{compose}(\mathrm{A}, \mathrm{B}, \mathrm{f}, i) \equiv \mathrm{f}: \mathrm{A} \rightarrow \mathrm{B}
\end{array}
$$

Proposition 3.6. If $\Xi \Longrightarrow 6\left[\right.$ is a rule such that $p$ is a pattern and $\mathrm{mv}(p)=|\Xi|_{\mathrm{obj}}$ then the rule is deterministic.

Proof. Consider a judgement $\Theta ; \Gamma \vdash b^{\prime} \in$, and instantiations $J$ and $K$ of $\Xi$ over $\Theta ; \Gamma$ such that $J_{*} p=K_{*} p=e$. Then $J$ and $K$ agree on object metavariables because they all appear in $p$, and on equational metavariables because they must.

We shall use patterns to find matching instantiations, when they exist. For this purpose we define the following notation. 
Definition 3.7. Given $\Xi$, a pattern $p$ over $\Xi$ such that $\operatorname{mv}(p)=|\Xi|_{\text {obj }}$, and an expression $e$ over $\Theta ; \Gamma$, we write

$$
\Xi \vdash p \triangleright t \rightsquigarrow I \quad \text { and } \quad \Xi \vdash p \triangleright t \not \leftrightarrow
$$

respectively when $I$ is an instantiation of $\Xi$ over $\Theta ; \Gamma$ such that $I_{*} p=t$, and when there is no such instantiation.

The reader should convince themselves that there is an obvious algorithm that computes from $\Xi, p$ and $t$ the unique $I$ such that $\Xi \vdash p \triangleright t \rightsquigarrow I$, or decides that it does not exist.

Rules are used not only to derive judgements, but also to invert derivable judgements to their premises, for the purpose of analyzing them. For example, when a term is normalized, we decide what steps to take by observing its structure, which amounts to applying an inversion principle, such as Theorem 2.21. In general, we may invert a derivable judgement $\Theta ; \Gamma \vdash j^{\prime}$ using a rule

$$
R=\left(\mathrm{M}_{1}: \mathcal{B}_{1}, \ldots, \mathrm{M}_{n}: \mathcal{B}_{n} \Longrightarrow j\right)
$$

by finding a derivable instantiation $I=\left\langle\mathrm{M}_{1} \mapsto e_{1}, \ldots, \mathrm{M}_{n} \mapsto e_{n}\right\rangle$ of its premises over $\Theta ; \Gamma$ such that $I_{*} j=j^{\prime}$. If $I$ is found, the judgement can be derived using the instantiation $I_{*} R$,

$$
\frac{\Theta ; \Gamma \vdash\left(I_{(k) *} \mathscr{B}_{k}\right) e_{k} \text { for } k=1, \ldots, n}{\Theta ; \Gamma \vdash I_{*} j}
$$

Under favorable conditions, it may happen that some of the above premises are known to be derivable ahead of time, so there is no need to rederive them. We are particularly interested in the case where all the object premises are of this kind.

Definition 3.8. Let $\Xi=\left[\mathrm{M}_{1}: \mathscr{B}_{1}, \ldots, \mathrm{M}_{n}: \mathscr{B}_{n}\right]$ be a metavariable context whose equational metavariables are $\mathrm{M}_{i_{1}}, \ldots, \mathrm{M}_{i_{m}}$. Given an instantiation $I$ of $\Xi$ over $\Theta$; [] such that $|\Xi| \cap|\Theta|=\emptyset$, the equational residue $\Xi / I$ is the metavariable context

$$
\Xi / I=\left[\Theta, \mathrm{M}_{i_{1}}: I_{\left(i_{1}\right) *} \mathcal{B}_{i_{1}}, \ldots, \mathrm{M}_{i_{m}}: I_{\left(i_{m}\right) *} \mathcal{B}_{i_{m}}\right] .
$$

The residual instantiation $I^{r}$ of $\Xi$ over $\Xi / I$ and [] is defined by

$$
I^{r}\left(\mathrm{M}_{i}\right)= \begin{cases}I\left(\mathrm{M}_{i}\right) & \text { if } \mathrm{M}_{i} \in|\Xi|_{\mathrm{obj}}, \\ \widehat{\mathrm{M}}_{i} & \text { otherwise. }\end{cases}
$$

Definition 3.9. In a raw type theory, a derivable raw rule $R=(\Xi \Longrightarrow j)$ is object-invertible when the following holds: whenever $I$ instantiates $\Xi$ over $\Theta$; [], with $\vdash \Theta$ mctx and $|\Xi| \cap|\Theta|=\emptyset$, if $\Theta ;[] \vdash I_{*} j$ is derivable then so is the residual instantiation $I^{r}$.

Let us explain how object-invertible rules shall be used. Suppose $\Xi \Longrightarrow s: A$ is objectinvertible, $\Xi \Longrightarrow s \equiv t: A$ is derivable, $I$ instantiates $\Xi$ over $\Theta ; \Gamma$, and $\Theta ; \Gamma \vdash I_{*} s: I_{*} A$ is given. We would like to derive $\Theta ; \Gamma \vdash I_{*} s \equiv I_{*} t: I_{*} A$ so that we may rewrite $I_{*} s$ to $I_{*} t$. Thus we must verify that $I$ is derivable. By object-invertibility its object premises are derivable, so we only need to check its equational ones. The following proposition ensures that such a procedure is valid.

Proposition 3.10. Consider an object-invertible rule $\Xi \Longrightarrow j$ and an instantiation $I$ over $\Theta ; \Gamma$, such that $\Theta ; \Gamma \vdash I_{*} j$ is derivable. Then $I$ is derivable if, for every equational boundary $\mathscr{B}=\{\vec{x}: \vec{A}\} \mathfrak{b}$ in $\Xi$, the judgement $\Theta ; \Gamma \vdash\left(I_{*} \mathscr{B}\right)\{\vec{x}\} \star$ is derivable. 
Proof. Let $J$ be the promotion of $I$ to $(\Theta, \Gamma)$ and the empty context. Because the rule is object-invertible, $J^{r}$ is derivable. Next, we promote each judgement from the statement to

$$
(\Theta, \Gamma) ;[] \vdash\left(J_{*} \mathcal{B}\right)\{\vec{x}\} \star \text {. }
$$

and observe that $J=K \circ J^{r}$, where $K$ is the instantiation of $\Xi / J$ over $(\Theta, \Gamma)$ and [] defined by

$$
K(\mathrm{M})= \begin{cases}\widehat{\mathrm{M}} & \text { if } \mathrm{M} \in|(\Theta, \Gamma)|, \\ \{\vec{x}\} \star & \text { otherwise. }\end{cases}
$$

Because $J^{r}$ is derivable, and $K$ is derivable thanks to derivability of judgements (3.2), it follows that $J$ is derivable. Therefore, $I$ is derivable too.

Example 3.11. Let us demonstrate how equational residues are going to be used in rewriting. Suppose we have derived

$$
\Theta ;[] \vdash \operatorname{fst}\left(U_{1}, V_{1} \text {, pair }\left(U_{2}, V_{2}, u, v\right)\right): U_{1}
$$

and would like to apply the $\beta$-rule (3.1) to it, i.e., we would like to establish

$$
\Theta ;[] \vdash \operatorname{fst}\left(U_{1}, V_{1}, \operatorname{pair}\left(U_{2}, V_{2}, u, v\right)\right) \equiv u: U_{1}
$$

First, using Theorem 2.19, we extract from (3.1) the derivability of its left-hand side

$$
\begin{aligned}
& \vdash \mathrm{A}_{1} \text { type } \quad \vdash \mathrm{A}_{2} \text { type } \quad \vdash \mathrm{B}_{1} \text { type } \vdash \mathrm{B}_{2} \text { type } \\
& \frac{\vdash \mathrm{s}: \mathrm{A}_{2} \quad \vdash \mathrm{t}: \mathrm{B}_{2} \quad \vdash \mathrm{A}_{1} \equiv \mathrm{A}_{2} \text { by } \zeta \quad \vdash \mathrm{B}_{1} \equiv \mathrm{B}_{2} \text { by } \xi}{\vdash \mathrm{fst}\left(\mathrm{A}_{1}, \mathrm{~B}_{1}, \operatorname{pair}\left(\mathrm{A}_{2}, \mathrm{~B}_{2}, \mathrm{~s}, \mathrm{t}\right)\right): \mathrm{A}_{1}}
\end{aligned}
$$

where we labeled the equational premises with metavariables $\zeta$ and $\xi$. We may compare (3.5) with (3.3) to get a matching instantiation

$$
I=\left\langle\mathrm{A}_{1} \mapsto U_{1}, \mathrm{~A}_{2} \mapsto U_{2}, \mathrm{~B}_{1} \mapsto V_{1}, \mathrm{~B}_{2} \mapsto V_{2}, \mathrm{~s} \mapsto u, \mathrm{t} \mapsto v, \zeta \mapsto \star, \xi \mapsto \star\right\rangle
$$

of its premises over $\Theta$; []. Now it would be a mistake to simply instantiate (3.1) with $I$ because the equational premises $\zeta$ and $\xi$ may not be derivable (the object premises are derivable by Theorem 2.19). However, because (3.5) is object-invertible by Corollary 3.17, proved below, the residual instantiation

$$
I^{r}=\left\langle\mathrm{A}_{1} \mapsto U_{1}, \mathrm{~A}_{2} \mapsto U_{2}, \mathrm{~B}_{1} \mapsto V_{1}, \mathrm{~B}_{2} \mapsto V_{2}, \mathrm{~s} \mapsto u, \mathrm{t} \mapsto v, \zeta \mapsto \zeta, \xi \mapsto \xi\right\rangle,
$$

is derivable. Hence, we may instantiate (3.1) with $I^{r}$ to derive

$$
\begin{aligned}
\Theta, \zeta:\left(U_{1} \equiv U_{2} \text { by } \square\right), \xi:\left(V_{1} \equiv V_{2} \text { by } \square\right) ;[] \vdash \\
\quad \operatorname{fst}\left(U_{1}, V_{1}, \operatorname{pair}\left(U_{2}, V_{2}, u, v\right)\right) \equiv u: U_{1} .
\end{aligned}
$$

Thus we must still verify $\Theta$; [] $\vdash U_{1} \equiv U_{2}$ and $\Theta$; [ $\vdash V_{1} \equiv V_{2}$, in order to conclude (3.4), precisely as expected.

Whether a rule is object-invertible depends not just on the rule itself, but on the ambient type theory too, for it may happen that $\Theta ; \Gamma \vdash I_{*} j$ is not derivable by the rule under consideration, but by another one that instantiates to the same conclusion.

Example 3.12. Consider the standard type theory whose specific rules are

$$
\overline{\vdash 0 \text { type }} \quad \overline{\vdash 1 \text { type }} \quad \overline{\vdash \mathrm{u}: 1} \quad \frac{\vdash \mathrm{v}: 1}{\vdash \mathrm{T}(\mathrm{v}) \text { type }} \quad \frac{\vdash \mathrm{e}: 0}{\vdash 0 \equiv 1}
$$


The derivable object rule

$$
\frac{\vdash \mathrm{e}: 0}{\vdash \mathrm{T}(\mathrm{e}) \text { type }}
$$

is not object-invertible, because the instantiation $I=\langle\mathrm{e} \mapsto \mathrm{u}\rangle$ yields the derivable judgement []; [] $\vdash \mathrm{T}(\mathrm{u})$ type, but []; [] $\vdash \mathrm{u}: 0$ is not derivable.

In the previous example the culprit is the application of term conversion to a metavariable. As it turns out, such conversions of variables are the principal obstruction to invertibility, so we define a syntactic property of judgements which prevents them.

Definition 3.13. An object judgement $\Theta ; \Gamma \vdash \mathcal{G}$ is natural for variables when the relation $\Theta ; \Gamma \vdash^{\natural} \mathscr{I}$ can be deduced using the rules in Figure 8.

$$
\begin{aligned}
& \frac{\Gamma(\mathrm{a})=A}{\Theta ; \Gamma \vdash^{\natural} \mathrm{a}: A} \quad \frac{\mathrm{a} \notin|\Gamma| \quad \Theta ; \Gamma, \mathrm{a}: A \vdash^{\natural} \mathcal{I}[\mathrm{a} / x]}{\Theta ; \Gamma \vdash^{\natural}\{x: A\} \mathcal{I}} \quad \frac{\begin{array}{c}
\Theta(\mathrm{M})=(\{\vec{x}: \vec{A}\} \square \text { type }) \\
\Theta ; \Gamma \vdash^{\natural} t_{i}: A\left[\vec{t}_{(i)} / \vec{x}_{(i)}\right] \text { for } i=1, \ldots, n
\end{array}}{\Theta ; \Gamma \vdash^{\natural} \mathrm{M}\left(t_{1}, \ldots, t_{n}\right) \text { type }} \\
& \Theta(\mathrm{M})=(\{\vec{x}: \vec{A}\} \square: B) \\
& \frac{\Theta ; \Gamma \vdash^{\natural} t_{i}: A\left[\vec{t}_{(i)} / \vec{x}_{(i)}\right] \text { for } i=1, \ldots, n}{\Theta ; \Gamma \vdash^{\natural} \mathrm{M}\left(t_{1}, \ldots, t_{n}\right): B[\vec{t} / \vec{x}]} \\
& \text { Rule for } \mathrm{S} \text { is } \mathrm{M}_{1}: \mathscr{B}_{1}, \ldots, \mathrm{M}_{n}: \mathscr { B } _ { n } \Longrightarrow b \longdiv { \mathrm { S } ( \widehat { M } _ { 1 } , \ldots , \widehat { M } _ { n } ) } \\
& I=\left\langle\mathrm{M}_{1} \mapsto e_{1}, \ldots, \mathrm{M}_{n} \mapsto e_{n}\right\rangle \\
& \frac{\Theta ; \Gamma \vdash^{\natural}\left(I_{(i) *} \mathscr{B}_{i}\right)\left[e_{i}\right] \quad \text { if } \mathscr{B}_{i} \text { is an object boundary }}{\Theta ; \Gamma \vdash^{\natural} \mathfrak{b}^{\prime} \mathrm{S}\left(e_{1}, \ldots, e_{n}\right)}
\end{aligned}
$$

Figure 8: Object judgements that are natural for variables

The point of this definition is that a derivable judgement which is natural for variables has a derivation in which any application of TT-META and TT-VAR is not immediately followed by a conversion, unless it appears in a subderivation of an equality judgement. The claim is established by a straightforward induction on the derivation of $\Theta ; \Gamma \vdash^{\natural} \mathcal{G}$ with the help of Theorem 2.21 .

The obvious pattern-matching algorithm scans a pattern and compares it to a term. It instantiates metavariables one by one and possibly out of order, which results in a chain of instantiations, each of which instantiates just one metavariable. Let us study such instantiations.

Definition 3.14. Let $\Xi=\left[\mathrm{M}_{1}: \mathcal{B}_{1}, \ldots, \mathrm{M}_{n}: \mathscr{B}_{n}\right]$ be a metavariable context, and $e$ an argument over $\Xi_{(k)}$ and the empty context with $\operatorname{ar}(e)=\operatorname{ar}\left(\mathscr{B}_{k}\right)$. The basic instantiation $\mathbb{I}\left(\Xi, \mathrm{M}_{k}, e\right)$ is defined by

$$
\mathbb{I}\left(\Xi, M_{k}, e\right)\left(M_{i}\right)= \begin{cases}\widehat{M}_{i} & \text { if } M_{k} \neq M_{i} \\ e & \text { if } M_{k}=M_{i}\end{cases}
$$

It is an instantiation of $\Xi$ over the metavariable context

$$
\mathbb{E}\left(\Xi, \mathrm{M}_{k}, e\right)=\left[\mathrm{M}_{1}: \mathscr{B}_{1}^{\prime}, \ldots, \mathrm{M}_{k-1}: \mathscr{B}_{k-1}^{\prime}, \mathrm{M}_{k+1}: \mathscr{B}_{k+1}^{\prime}, \ldots, \mathrm{M}_{n}: \mathscr{B}_{n}^{\prime}\right]
$$

and the empty context, where $\mathscr{B}_{j}^{\prime}=\mathbb{I}\left(\Xi, \mathrm{M}_{k}, e\right)_{(j) *} \mathcal{B}_{j}$. 
Lemma 3.15. A basic instantiation $\mathbb{I}\left(\Xi, \mathrm{M}_{k}, e\right)$ is derivable if $\vdash \Xi \operatorname{mctx}$ and $\Xi_{(k)} \vdash \mathscr{B}_{k}[e$, in which case $\vdash \mathbb{E}\left(\Xi, \mathrm{M}_{k}, e\right)$ mctx also holds.

Proof. For $i<k$, the judgement $\mathbb{E}\left(\Xi, \mathrm{M}_{k}, e\right) \vdash\left(\mathbb{I}\left(\Xi, \mathrm{M}_{k}, e\right)_{(i) *} \mathcal{B}_{i}\right) \widehat{\mathrm{M}}_{i}$ holds by abstraction and the metavariable rule, where we invert $\vdash \Xi$ mctx to validate the abstractions.

The judgement $\mathbb{E}\left(\Xi, \mathrm{M}_{k}, e\right) \vdash\left(\mathbb{I}\left(\Xi, \mathrm{M}_{k}, e\right)_{(k) *} \mathcal{B}_{k}\right) e$ follows by weakening from $\Xi_{(k)} \vdash \mathscr{B}_{k} e$ because $\mathbb{E}\left(\Xi, \mathrm{M}_{k}, e\right)_{(k)}=\Xi_{(k)}$.

For $i>k$, we again use abstraction and the metavariable rule, where abstractions are now validated by inversion of $\vdash \Xi$ mctx and Theorem 2.17 applied to $\mathbb{I}\left(\Xi, \mathrm{M}_{k}, e\right)_{(i)}$.

The derivation of $\vdash \mathbb{E}\left(\Xi, \mathrm{M}_{k}, e\right)$ mctx has two parts. First, $\mathbb{E}\left(\Xi, \mathrm{M}_{k}, e\right)_{(k)}$ coincides with $\Xi_{(k)}$ and so we just reuse $\vdash \Xi_{(k)}$ mctx. For $i>k$, we derive $\mathbb{E}\left(\Xi, \mathrm{M}_{k}, e\right)_{\left(\mathrm{M}_{i}\right)} \vdash \mathscr{B}_{i}^{\prime}$ as the instantiation of $\Xi_{(i)} \vdash \mathscr{B}_{i}$ by

$$
\mathbb{I}\left(\Xi, \mathrm{M}_{k}, e\right)_{(i)} \in \operatorname{Inst}\left(\Xi_{(i)}, \mathbb{E}\left(\Xi, \mathrm{M}_{k}, e\right)_{\left(\mathrm{M}_{i}\right)},[]\right),
$$

which is observed to be derivable.

We define particular compositions of chains of basic instantiations, as follows. Given a metavariable context $\Xi=\left[\mathrm{M}_{1}: \mathscr{B}_{1}, \ldots, \mathrm{M}_{n}: \mathcal{B}_{n}\right]$ and an instantiation

$$
I=\left\langle\mathrm{M}_{1} \mapsto e_{1}, \ldots, \mathrm{M}_{n} \mapsto e_{n}\right\rangle
$$

of $\Xi$ over $\Theta ;$ [], define the instantiation

$$
\mathbb{J}_{\Xi, \Theta, I}\left(\mathrm{M}_{i_{1}}, \ldots, \mathrm{M}_{i_{m}}\right) \in \operatorname{Inst}\left(\Xi, \mathbb{F}_{\Xi, \Theta, I}\left(\mathrm{M}_{i_{1}}, \ldots, \mathrm{M}_{i_{m}}\right),[]\right)
$$

and the metavariable context $\mathbb{F}_{\Xi, \Theta, I}\left(\mathrm{M}_{i_{1}}, \ldots, \mathrm{M}_{i_{m}}\right)$ by

$$
\begin{aligned}
& \mathbb{F}_{\Xi, \Theta, I}()=\langle\Theta, \Xi\rangle \\
& \mathbb{J}_{\Xi, \Theta, I}()=\left\langle\mathrm{M}_{1} \mapsto \widehat{\mathrm{M}}_{1}, \ldots, \mathrm{M}_{n} \mapsto \widehat{\mathrm{M}}_{n}\right\rangle \\
& \mathbb{F}_{\Xi, \Theta, I}\left(\mathrm{M}_{i_{1}}, \ldots, \mathrm{M}_{i_{m+1}}\right)=\mathbb{E}\left(\mathbb{F}_{\Xi, \Theta, I}\left(\mathrm{M}_{i_{1}}, \ldots, \mathrm{M}_{i_{m}}\right), \mathrm{M}_{i_{m+1}}, e_{i_{m+1}}\right) \\
& \mathbb{J}_{\Xi, \Theta, I}\left(\mathrm{M}_{i_{1}}, \ldots, \mathrm{M}_{i_{m+1}}\right)=\mathbb{I}\left(\mathbb{F}_{\Xi, \Theta, I}\left(\mathrm{M}_{i_{1}}, \ldots, \mathrm{M}_{i_{m}}\right), \mathrm{M}_{i_{m+1}}, e_{i_{m+1}}\right) \circ \\
& \mathbb{J}_{\Xi, \Theta, I}\left(\mathrm{M}_{i_{1}}, \ldots, \mathrm{M}_{i_{m}}\right)
\end{aligned}
$$

In the above definition we require $|\Xi| \cap|\Theta|=\emptyset$ and that $\mathrm{M}_{i_{1}}, \ldots, \mathrm{M}_{i_{m}}$ are all distinct. We elide the subscripts and write $\mathbb{J}\left(\mathrm{M}_{i_{1}}, \ldots, \mathrm{M}_{i_{m}}\right)$ and $\mathbb{F}\left(\mathrm{M}_{i_{1}}, \ldots, \mathrm{M}_{i_{m}}\right)$ when no confusion can arise. A straightforward induction shows that

$$
\mathbb{F}_{\Xi, \Theta, I}\left(\mathrm{M}_{i_{1}}, \ldots, \mathrm{M}_{i_{m}}\right)\left(\mathrm{M}_{j}\right)=\mathbb{J}_{\Xi, \Theta, I}\left(\mathrm{M}_{i_{1}}, \ldots, \mathrm{M}_{i_{m}}\right)_{*} \mathcal{B}_{j}
$$

for any $\mathrm{M}_{j} \in|\Xi| \backslash\left\{\mathrm{M}_{i_{1}}, \ldots, \mathrm{M}_{i_{m}}\right\}$. The instantiation $\mathbb{J}_{\Xi, \Theta, I}\left(\mathrm{M}_{i_{1}}, \ldots, \mathrm{M}_{i_{m}}\right)$ plays a role in proving object-invertibility, because $\left\{\mathrm{M}_{i_{1}}, \ldots, \mathrm{M}_{i_{m}}\right\}=|\Xi|_{\text {obj }}$ implies

$$
\mathbb{J}_{\Xi, \Theta, I}\left(\mathrm{M}_{i_{1}}, \ldots, \mathrm{M}_{i_{m}}\right)=I^{r} \quad \text { and } \quad \mathbb{F}_{\Xi, \Theta, I}\left(\mathrm{M}_{i_{1}}, \ldots, \mathrm{M}_{i_{m}}\right)=\Xi / I \text {. }
$$

We are now in possession of all the ingridients necessary to relate pattern matching and object-invertibility. Recall that, given a rule $\Xi \Longrightarrow b p$ whose head is a pattern $p$ and an instantiation $I$ of $\Xi$ over $\Theta$; [], we would like to show that $I^{r}$ is derivable. The following lemma, whose purpose we explain shortly, is a stepping stone to accomplishing the goal.

Lemma 3.16. In a standard type theory, let $\Xi \Longrightarrow b \mid p$ be a derivable object rule which is natural for variables, $p$ a pattern, and $I$ an instantiation of $\Xi=\left[\mathrm{M}_{1}: \mathcal{B}_{1}, \ldots, \mathrm{M}_{n}: \mathcal{B}_{n}\right]$ over $\Theta ;[]$ such that $|\Xi| \cap|\Theta|=\emptyset$, and $\Theta ;[] \vdash I_{*}(b[p)$ is derivable. 
Suppose $\vec{N}=\left(N_{1}, \ldots, N_{m}\right)$ is a sequence of distinct metavariables, $\left\{N_{1}, \ldots, N_{m}\right\} \subseteq|\Xi|$, $\operatorname{mv}(\mathcal{B}) \subseteq\left\{N_{1}, \ldots, N_{m}\right\} \cup \operatorname{mv}(p)$, and both $\vdash \mathbb{F}_{\Theta, \Xi, I}(\vec{N}) \operatorname{mctx}$ and $\mathbb{J}_{\Theta, \Xi, I}(\vec{N})$ are derivable. Then $\vec{N}$ can be extended to a sequence of distinct metavariables $\vec{N}^{\prime}=\left(N_{1}, \ldots, N_{\ell}\right)$ such that $\left\{N_{1}, \ldots, N_{\ell}\right\}=\left\{N_{1}, \ldots, N_{m}\right\} \cup \operatorname{mv}(p)$, and both $\vdash \mathbb{F}\left(\vec{N}^{\prime}\right) \operatorname{mctx}$ and $\mathbb{J}\left(\vec{N}^{\prime}\right)$ are derivable.

Let us explain how the lemma shall be used. As noted above, $I^{r}$ coincides with $\mathbb{J}(\vec{N})$ when $\vec{N}$ lists all of $|\Xi|_{\text {obj }}$. Therefore, we may establish derivability of $I^{r}$ by starting with $\vec{N}=()$ and extending it with metavariables until it encompasses $|\Xi|_{\text {obj }}$. The lemma guarantees that one such extension step can be done with the aid of patterns in a way that preserves derivability of $\mathbb{J}(\vec{N})$.

Proof of Lemma 3.16. Let $I=\left\langle\mathrm{M}_{1} \mapsto e_{1}, \ldots, \mathrm{M}_{n} \mapsto e_{n}\right\rangle$. We proceed by induction on the structure of $p$, and elide the subscripts to keep the notation shorter.

Case $p=\mathrm{M}_{k}, \mathscr{B}_{k}=(\square$ type $)$, and $\boldsymbol{b}=\left(\square\right.$ type): If $\mathrm{M}_{k}$ appears in $\vec{N}$ we let $\ell=m$ and we are done. Otherwise we set $\ell=m+1$ and $N_{m+1}=\mathrm{M}_{k}$. Because composition of derivable instantiations is derivable, we only need to show that $\mathbb{I}\left(\mathbb{F}(\vec{M}), \mathrm{M}_{k}, e_{k}\right)$ is derivable, which by Lemma 3.15 reduces to

which equals

$$
\mathbb{F}(\vec{N})_{\left(\mathrm{M}_{k}\right)} ;[] \vdash\left(\mathbb{J}(\vec{N})_{*} \mathscr{B}_{k}\right) e_{k},
$$

$$
\mathbb{F}(\vec{N})_{\left(\mathrm{M}_{k}\right)} ;[] \vdash e_{k} \text { type. }
$$

It is derivable by weakening from the assumption $\Theta$; [ $\vdash e_{k}$ type.

Case $p=\mathrm{S}\left(q_{1}, \ldots, q_{m}\right)$ : Suppose the symbol rule for $\mathrm{S}$ is

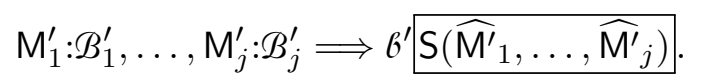

By applying Corollary 2.24 to $\Xi ;[] \vdash b[S(\vec{q})]$ and letting $K=\left[\mathrm{M}_{1}^{\prime} \mapsto q_{1}, \ldots, \mathrm{M}_{j}^{\prime} \mapsto q_{j}\right]$, we obtain for $i=1, \ldots, j$ derivations of

$$
\Xi ;[] \vdash\left(K_{(i) *} \mathcal{B}_{i}^{\prime}\right) q_{i} \text {. }
$$

Similarly, from derivability of $\Theta ;[] \vdash\left(I_{*} b\right) \mathrm{S}\left(I_{*} \vec{q}\right)$ we obtain derivability of

$$
\Theta ;[] \vdash\left(\left(I_{*} K\right)_{(i) *} \mathcal{B}_{i}^{\prime}\right) I_{*} q_{i},
$$

which is equal to

$$
\Theta ;[] \vdash I_{*}\left(\left(K_{(i) *} \mathcal{B}_{i}^{\prime}\right) q_{i}\right) .
$$

We define $\vec{L}_{0}, \ldots, \vec{L}_{j}$ such that $\vec{L}_{0}=\vec{N}$, and for $i=1, \ldots, j$, the sequence $\vec{L}_{i}$ extends $\vec{L}_{i-1}$ by $\operatorname{mv}\left(q_{i}\right)$, and both $\vdash \mathbb{F}\left(\vec{L}_{i}\right)$ mctx and $\mathbb{J}\left(\vec{L}_{i}\right)$ are derivable. We may then finish the proof by taking $\vec{N}^{\prime}=\vec{L}_{j}$. Assuming $\vec{L}_{i-1}$ has been constructed, we consider two cases.

First, if $q_{i}$ is a non-abstracted object pattern then we obtain $\vec{L}_{i}$ by applying the induction hypothesis to (3.7), (3.9) and $\vec{L}_{i-1}$. We may do so because $\operatorname{mv}\left(K_{(i) *} \mathscr{B}_{i}^{\prime}\right) \subseteq$ $\operatorname{mv}\left(q_{1}\right) \cup \cdots \cup \operatorname{mv}\left(q_{i-1}\right)$, which is contained in $\vec{L}_{i-1}$.

Second, if $q_{i}=\{\vec{x}\} \mathrm{M}_{k}(\vec{x})$ we proceed as follows. If $\mathrm{M}_{k}$ appears in $\vec{L}_{i-1}$, we take $\vec{L}_{i}=\vec{L}_{i-1}$ and we are done. Otherwise, we take $\vec{L}_{i}=\left(\vec{L}_{i-1}, \mathrm{M}_{k}\right)$. We need to show derivability of $\vdash \mathbb{F}\left(\vec{L}_{i}\right)$ mctx and $\mathbb{J}\left(\vec{L}_{i}\right)$. Because $\mathbb{J}\left(\vec{L}_{i}\right)=\mathbb{I}\left(\mathbb{F}\left(\vec{L}_{i-1}\right), \mathrm{M}_{k}, e_{k}\right) \circ \mathbb{J}\left(\vec{L}_{i-1}\right)$ and $\mathbb{J}\left(\vec{L}_{i-1}\right)$ is derivable it suffices to show that $\mathbb{I}\left(\mathbb{F}\left(\vec{L}_{i-1}\right), \mathrm{M}_{k}, e_{k}\right)$ is derivable, and therefore by Lemma 3.15 that

$$
\mathbb{F}\left(\vec{L}_{i-1}\right)_{\left(\mathrm{M}_{k}\right)} ;[] \vdash\left(\mathbb{J}\left(\vec{L}_{i-1}\right)_{*} \mathscr{B}_{k}\right) e_{k} .
$$


We claim that (3.10) is just a weakening of (3.8). Obviously, $\mathbb{F}\left(\vec{L}_{i-1}\right)_{\left(\mathrm{M}_{k}\right)}$ extends $\Theta$ and $I_{*} q_{i}=e_{k}$. It remains to be seen that $\mathbb{J}\left(\vec{L}_{i-1}\right)_{*} \mathscr{B}_{k}$ and $\left(I_{*} K\right)_{(i) *} \mathscr{B}_{i}^{\prime}$ are the same. The judgement (3.7) equals $\Xi ;[] \vdash\left(K_{(i) *} \mathscr{B}_{i}^{\prime}\right)\{\vec{x}\} \mathrm{M}_{k}(\vec{x})$. By the naturality-for-variables assumption it is derivable without conversions, which is only possible if $K_{(i) *} \mathscr{B}_{i}^{\prime}$ is $\mathscr{B}_{k}$. Therefore,

$$
\left(I_{*} K\right)_{(i) *} \mathcal{B}_{i}^{\prime}=I_{*}\left(K_{(i) *} \mathscr{B}_{i}^{\prime}\right)=\mathbb{J}\left(\vec{L}_{i-1}\right)_{*}\left(K_{(i) *} \mathcal{B}_{i}^{\prime}\right)=\mathbb{J}\left(\vec{L}_{i-1}\right)_{*} \mathcal{B}_{k},
$$

where the second step is valid because $\operatorname{mv}\left(K_{(i) *} \mathcal{B}_{i}^{\prime}\right) \subseteq \operatorname{mv}\left(q_{1}\right) \cup \cdots \cup \operatorname{mv}\left(q_{i-1}\right)$, which is contained in $\vec{L}_{i-1}$.

Corollary 3.17. In a standard type theory, consider a derivable finitary object rule $\Xi \Longrightarrow b p$ which is natural for variables. If $p$ is a pattern and $\operatorname{mv}(p)=|\Xi|_{\text {obj }}$ then the rule is objectinvertible.

Proof. Consider an instantiation $I$ of $\Xi$ over $\Theta$; [], such that $\vdash \Theta$ mctx and $\Theta$; [] $\vdash\left(I_{*} b\right) I_{*} p$ are derivable. Without loss of generality we may assume $|\Xi| \cap|\Theta|=\emptyset$.

We apply Lemma 3.16 with the empty sequence $\vec{N}=()$, noting that $\operatorname{mv}(\mathfrak{b}) \subseteq \operatorname{mv}(p)$, that $\mathbb{F}()=\langle\Theta, \Xi\rangle$ and that $\vdash\langle\Theta, \Xi\rangle$ mctx is derivable because the rule is finitary and we assumed $\vdash \Theta$ mctx. This way we obtain a sequence $\vec{N}^{\prime}=\left(N_{1}^{\prime}, \ldots, N_{\ell}^{\prime}\right)$ such that $\operatorname{mv}(p)=\left\{N_{1}^{\prime}, \ldots, N_{\ell}^{\prime}\right\}$ and $\mathbb{J}\left(\vec{N}^{\prime}\right)$ is derivable. Because $\operatorname{mv}(p)=|\Xi|_{\text {obj }}$, it follows that $\mathbb{J}\left(\vec{N}^{\prime}\right)$ coincides with $I^{r}$, hence it is derivable too.

3.2. Computation and extensionality rules. The equality checking algorithm uses two kinds of equational rules, which we describe here and prove that they have the desired properties. First, we have the rules that govern normalization.

Definition 3.18. A derivable finitary rule $\Theta \Longrightarrow A \equiv B$ is a type computation rule if $\Theta \Longrightarrow A$ type is deterministic and object-invertible.

Definition 3.19. A derivable finitary rule $\Theta \Longrightarrow u \equiv v: A$ is a term computation rule if $u$ is a term symbol application and $\Theta \Longrightarrow u: \tau_{\Theta ;[]}(u)$ is deterministic and object-invertible.

The reason behind the first condition in the definition of a term computation rule is that for term symbol applications Proposition 2.23 holds, which is needed in the proof of soundness (Theorem 3.26). We exhibit in Example 3.25 what can go wrong if we allow for a metavariable as the lefthand-side of the equation. One might hope that the second condition in Definition 3.19 could be relaxed to $\Theta \Longrightarrow u: A$. However, the additional flexibility is only apparent, for if a term has a type then it has the natural type as well. In any case, in the proofs of soundness (Theorems 3.26 and 3.27) we rely on having the natural type.

A computation rule may be recognized using the following criterion.

Proposition 3.20. In a standard type theory:

(1) A derivable finitary rule $\Xi \Longrightarrow P \equiv B$ is a type computation rule if $P$ is a type pattern, $\mathrm{mv}(P)=|\Xi|_{\mathrm{obj}}$, and $\Xi \Longrightarrow P$ type is natural for variables.

(2) A derivable finitary rule $\Xi \Longrightarrow p \equiv v: A$ is a term computation rule if $p$ is a term pattern, $\operatorname{mv}(p)=|\Xi|_{\mathrm{obj}}$, and $\Xi \Longrightarrow p: \tau_{\Xi ;[]}(p)$ is natural for variables.

Proof. The rule $\Xi \Longrightarrow P$ type is derivable by Theorem 2.19 , and $\Xi \Longrightarrow p: \tau_{\Xi ;]}(p)$ by Theorem 2.19 and Corollary 2.25. Observe that $\operatorname{mv}(P)=|\Xi|_{\mathrm{obj}}$ and $\operatorname{mv}(p)=|\Xi|_{\mathrm{obj}}$, and apply Proposition 3.6 and Corollary 3.17 respectively to $\Xi \Longrightarrow P$ type and to $\Xi \Longrightarrow p: \tau_{\Xi ;[]}(p)$. 
Example 3.21. Typical $\beta$-rules satisfy the conditions of Proposition 3.20, after their lefthand sides have been linearized, as in Example 3.4. Another example is the $\beta$-rule for application

$$
\frac{\vdash \text { A type } \quad \vdash\{x: \mathrm{A}\} \text { B type } \quad \vdash\{x: \mathrm{A}\} \mathrm{s}: \mathrm{B}(x) \quad \vdash \mathrm{t}: \mathrm{A}}{\vdash \operatorname{apply}(\mathrm{A},\{x\} \mathrm{B}(x), \lambda(\mathrm{A},\{x\} \mathrm{B}(x),\{x\} \mathrm{s}(x)), \mathrm{t}) \equiv \mathrm{s}(\mathrm{t}): \mathrm{B}(\mathrm{t})}
$$

whose linearized form is

$$
\begin{array}{cc}
\vdash \mathrm{A}_{1} \text { type } & \vdash\left\{x: \mathrm{A}_{1}\right\} \mathrm{B}_{1} \text { type } \\
\vdash \mathrm{A}_{2} \text { type } & \vdash\left\{x: \mathrm{A}_{2}\right\} \mathrm{B}_{2} \text { type } \\
\vdash\left\{x: \mathrm{A}_{2}\right\} \mathrm{s}: \mathrm{B}_{2}(x) \quad \vdash \mathrm{t}: \mathrm{A}_{1} \\
\vdash \mathrm{A}_{1} \equiv \mathrm{A}_{2} \vdash\left\{x: \mathrm{A}_{1}\right\} \mathrm{B}_{1}(x) \equiv \mathrm{B}_{2}(x) \\
\hline \operatorname{apply}\left(\mathrm{A}_{1},\{x\} \mathrm{B}_{1}(x), \lambda\left(\mathrm{A}_{2},\{x\} \mathrm{B}_{2}(x),\{x\} \mathrm{s}(x)\right), \mathrm{t}\right) \equiv \mathrm{s}(\mathrm{t}): \mathrm{B}_{1}(\mathrm{t})
\end{array}
$$

which satisfies Proposition 3.20.

We also allow the somewhat unusual rule

$$
\overline{\vdash U \text { type }} \quad \frac{\vdash A \text { type }}{\vdash A \equiv U}
$$

because it allows us to dispense with all questions about equality of types in case we want to work with an uni-typed theory (some would call it untyped).

The second kind of rules is used by the algorithm to reduce an equation to subordinate equations by matching its type.

Definition 3.22. An extensionality rule is a derivable finitary rule of the form

$$
\Theta, \mathrm{s}:(\square: A), \mathrm{t}:(\square: A), \Phi \Longrightarrow \mathrm{s} \equiv \mathrm{t}: A
$$

such that $\Phi$ contains only equational premises, and $\Theta \Longrightarrow A$ type is deterministic and object-invertible.

An extensional rule may be recognized with the following criterion.

Proposition 3.23. In a standard type theory, a derivable finitary rule of the form

$$
\Xi, \mathrm{s}:(\square: P), \mathrm{t}:(\square: P), \Phi \Longrightarrow \mathrm{s} \equiv \mathrm{t}: P
$$

is an extensionality rule if $\Phi$ contains only equational premises, $P$ is a type pattern, $\operatorname{mv}(P)=$ $|\Xi|_{\mathrm{obj}}$, and $\Xi \Longrightarrow P$ type is natural for variables.

Proof. Apply Proposition 3.6 and Corollary 3.17 to $\Xi \Longrightarrow P$ type.

Extensionality rules that one finds in practice typically satisfy the above syntactic condition, even without linearization. Here are a few.

Example 3.24. Extensionality rules typically state that elements of a type are equal when their parts are equal. For example, extensionality for simple products states that pairs are equal if their components are equal:

$$
\begin{array}{ccc}
\vdash \text { A type } \quad \vdash \mathrm{B} \text { type } & \vdash \mathrm{s}: \mathrm{A} \times \mathrm{B} \quad \vdash \mathrm{t}: \mathrm{A} \times \mathrm{B} \\
\vdash \mathrm{fst}(\mathrm{A}, \mathrm{B}, \mathrm{s}) \equiv \mathrm{fst}(\mathrm{A}, \mathrm{B}, \mathrm{t}): \mathrm{A} & \vdash \operatorname{snd}(\mathrm{A}, \mathrm{B}, \mathrm{s}) \equiv \operatorname{snd}(\mathrm{A}, \mathrm{B}, \mathrm{t}): \mathrm{B} \\
\hline & \vdash \mathrm{s} \equiv \mathrm{t}: \mathrm{A} \times \mathrm{B}
\end{array}
$$


Similarly, the extensionality rule for dependent functions states that they are equal if their generic applications are equal:

$$
\begin{gathered}
\vdash \text { A type } \quad \vdash\{x: \mathrm{A}\} \mathrm{B} \text { type } \quad \vdash \mathrm{s}: \Pi(\mathrm{A},\{x\} \mathrm{B}(x)) \quad \vdash \mathrm{t}: \Pi(\mathrm{A},\{x\} \mathrm{B}(x)) \\
\vdash\{x: \mathrm{A}\} \operatorname{apply}(\mathrm{A},\{x\} \mathrm{B}(x), \mathrm{s}, \mathrm{x}) \equiv \operatorname{apply}(\mathrm{A},\{x\} \mathrm{B}(x), \mathrm{t}, \mathrm{x}): \mathrm{B}(x) \\
\vdash \mathrm{s} \equiv \mathrm{t}: \Pi(\mathrm{A},\{x\} \mathrm{B}(x))
\end{gathered}
$$

The above is not to be confused with propositional function extensionality, which is a certain term that maps point-wise propositional equality of functions to their propositional equality.

Example 3.25. Some extensionality rules have no equational premises. The first one that comes to mind is the rule stating that all elements of the unit type are equal:

$$
\frac{\vdash \mathrm{s}: \text { unit } \quad \vdash \mathrm{t}: \text { unit }}{\vdash \mathrm{s} \equiv \mathrm{t}: \text { unit }}
$$

The corresponding $\eta$-rule ( $\star$ is the canonical inhabitant of unit)

$$
\frac{\vdash \mathrm{t}: \text { unit }}{\vdash \mathrm{t} \equiv \star}
$$

cannot be incorporated as a computation rule naively because the bare metavariable on the left-hand side matches any term, even if its type is not (judgementally equal to) unit. Since our normalization procedure in Section 3.3 does not check for equality of types separately, such rules do not behave well as computation rules. Another rule of this kind is the judgemental variant of Uniqueness of Identity Proofs (UIP) which equates any two proofs of a propositional identity:

$$
\frac{\vdash \text { A type } \quad \vdash \mathrm{a}: \mathrm{A} \quad \vdash \mathrm{b}: \mathrm{A} \quad \vdash \mathrm{p}: \operatorname{Id}(\mathrm{A}, \mathrm{a}, \mathrm{b}) \quad \vdash \mathrm{q}: \operatorname{Id}(\mathrm{A}, \mathrm{a}, \mathrm{b})}{\vdash \mathrm{p} \equiv \mathrm{q}: \operatorname{Id}(\mathrm{A}, \mathrm{a}, \mathrm{b})}
$$

The corresponding $\eta$-rule is as troublesome as the one for unit:

$$
\frac{\vdash \text { A type } \quad \vdash \mathrm{a}: \mathrm{A} \quad \vdash \mathrm{p}: \operatorname{Id}(\mathrm{A}, \mathrm{a}, \mathrm{a})}{\vdash \mathrm{p} \equiv \operatorname{refl}(\mathrm{A}, \mathrm{a}): \operatorname{Id}(\mathrm{A}, \mathrm{a}, \mathrm{a})}
$$

The principle has been used, for example, in the cubical type theory XTT for Bishop sets [SAG20].

Here is one last example:

$$
\frac{\vdash \text { A type }}{\vdash\|\mathrm{A}\| \text { type }} \quad \frac{\vdash \text { A type } \vdash \mathrm{t}: \mathrm{A}}{\vdash|\mathrm{t}|:\|\mathrm{A}\|} \quad \frac{\vdash \text { A type } \vdash \mathrm{u}:\|\mathrm{A}\| \quad \vdash \mathrm{v}:\|\mathrm{A}\|}{\vdash \mathrm{u} \equiv \mathrm{v}:\|\mathrm{A}\|}
$$

The above rules describe a kind of "judgemental truncation", which is like the propositional truncation from homotopy type theory, except that it equates all terms of $\|A\|$ judgementally. It is unclear what elimination rule of judgemental truncation would be, but one is reminded of the proof-irrelevant propositions [GCST19]. 
3.3. Principal arguments and normalization. Normalization rewrites an expression $\mathrm{S}\left(e_{1}, \ldots, e_{n}\right)$ by normalizing some of the arguments $e_{1}, \ldots, e_{n}$, applying a computation rule, and repeating the process. We say that an argument $e_{i}$ (or more precisely, its position $i$ ) is principal for $S$ if it is so normalized. By varying the selection of principal arguments we may control the algorithm to compute various kinds of normal form. For example, in $\lambda$-calculus the weak-head normal form is obtained when the only principal argument is the head of an application, while taking all arguments to be principal leads to the strong normal form. Our algorithm is flexible in this regard, as it takes the information about principality of arguments as input. In Section 4.1 we discuss how appropriate principal arguments can be chosen.

In specific cases normal forms are characterized by their syntactic structure, for example a normal form in the $\lambda$-calculus is an expression without $\beta$-redeces. One then proves that the normalization procedure always leads to a normal form. We are faced with a general situation in which no such syntactic characterization is available. Luckily, the algorithm never needs to recognize normal forms, although we do have to keep track of which expressions have already been subjected to the normalization procedure, so that we avoid normalizing them again.

Normalization is parametrized by the following data:

(1) a standard type theory $T$,

(2) a family $C$ of computation rules for $T$ (Definitions 3.18 and 3.19),

(3) for each symbol S taking $k$ arguments, a set $\wp(\mathrm{S}) \subseteq\{1, \ldots, k\}$ of its principal arguments,

It has three interdependent variations:

$$
\begin{aligned}
& \Theta ; \Gamma \vdash \mathcal{B} e e^{\prime} \\
& \Theta ; \Gamma \vdash b \mathcal{S}(\vec{e}) \triangleright_{\mathrm{p}} \mathrm{S}\left(\vec{e}^{\prime}\right) \\
& \Theta ; \Gamma \vdash b e \triangleright_{\mathrm{c}} e^{\prime}
\end{aligned}
$$

normalize argument $e$ to $e^{\prime}$, normalize the principal arguments of $\mathrm{S}$, use a computation rule to rewrite $e$ to $e^{\prime}$.

Specifically,

$$
\Theta ; \Gamma \vdash\left(A \triangleright A^{\prime}\right) \text { type } \quad \text { and } \quad \Theta ; \Gamma \vdash t \triangleright t^{\prime}: B
$$

respectively express the facts that the type $A$ normalizes to $A^{\prime}$ and the term $t$ to $t^{\prime}$. Figure 9 specifies the normalization procedure. Note that normalization is mutually recursive with equality checking, because the rule for $\triangleright_{c}$ resolves equational premises using equational checking from Figure 10. We omitted the clauses for metavariable applications, as they are analogous to symbol applications. That is, for the purposes of normalization and equality checking, an object metavariable $\mathrm{M}$ with boundary $\mathcal{B}$ and arity $\operatorname{ar}(\mathscr{B})=(c, n)$ is construed as a primitive symbol of syntactic class $c$ taking $n$ term arguments.

Normalization of arguments is syntax-directed and deterministic, and so is normalization of principal arguments. However, the applications of computation rules need not terminate, and the computation rules may be a source of non-determinism when several apply to the same expression. We discuss strategies for dealing with these issues in Section 4.2. 


$$
\begin{aligned}
& \left(\Xi \Longrightarrow b^{\prime} p \equiv v\right) \in C \quad \Xi \vdash p \triangleright s \rightsquigarrow I \\
& \Theta ; \Gamma \vdash I_{*}\left(\mathscr{B} e \sim e^{\prime}\right) \text { for }\left(\mathrm{M}: \mathscr{B} e \equiv e^{\prime} \text { by } \square\right) \in \Xi \\
& \Theta ; \Gamma \vdash b s \triangleright_{c} I_{*} v \\
& \text { Rule for } \mathrm{S} \text { is } \mathrm{M}_{1}: \mathscr{B}_{1}, \ldots, \mathrm{M}_{n}: \mathscr{B}_{n} \Longrightarrow \mathcal{G}^{\prime} \widehat{\mathrm{S}\left(\widehat{\mathrm{M}}_{1}, \ldots, \widehat{\mathrm{M}}_{n}\right)} \\
& \Theta ; \Gamma \vdash\left(\left\langle\mathrm{M}_{1} \mapsto e_{1}, \ldots, \mathrm{M}_{i-1} \mapsto e_{i-1}\right\rangle \mathscr{B}_{i}\right) e_{i} \triangleright e_{i}^{\prime} \quad \text { if } i \in \wp(\mathrm{S}) \\
& e_{i}=e_{i}^{\prime} \quad \text { if } i \notin \wp(\mathrm{S}) \\
& \Theta ; \Gamma \vdash b \longdiv { \mathrm { S } ( \vec { e } ) \triangleright _ { \mathrm { p } } \mathrm { S } ( \vec { e } ^ { \prime } ) }
\end{aligned}
$$

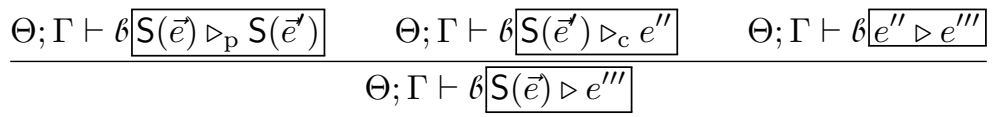

$$
\begin{aligned}
& \Theta ; \Gamma \vdash b \mathrm{~S}(\vec{e}) \triangleright_{\mathrm{p}} \mathrm{S}(\vec{e}) \\
& \frac{\Xi \vdash p \triangleright \mathrm{S}\left(\vec{e}^{\prime}\right) \not \rightarrow \quad \text { for }\left(\Xi \Longrightarrow b^{\prime} \mid p \equiv v\right) \in C}{\Theta ; \Gamma \vdash b\left[\mathrm{~S}(\vec{e}) \triangleright \mathrm{S}\left(\vec{e}^{\prime}\right)\right]} \quad \frac{\mathrm{a} \notin|\Gamma| \quad \Theta ; \Gamma, \mathrm{a}: A \vdash(\mathscr{B}[\mathrm{a} / x]) e[\mathrm{a} / x] \triangleright e^{\prime}}{\Theta ; \Gamma \vdash\{x: A\} \mathscr{B}\left[\{x\} e \triangleright\{x\} e^{\prime}[x / \mathrm{a}]\right.}
\end{aligned}
$$

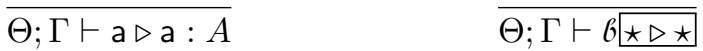

Figure 9: Normalization with computation rules $C$ and principal arguments $\wp$.

3.4. Type-directed and normalization phases. We are finally ready to describe equality checking, which is performed by several mutually recursive phases:

$$
\begin{array}{ll}
\Theta ; \Gamma \vdash \mathscr{B} e \sim e^{\prime} & e \text { and } e^{\prime} \text { are equal arguments } \\
\Theta ; \Gamma \vdash s \sim_{\mathrm{e}} t: A & s \text { and } t \text { are extensionally equal } \\
\Theta ; \Gamma \vdash s \sim_{\mathrm{n}} t: A & \text { normalized terms } s \text { and } t \text { are equal } \\
\Theta ; \Gamma \vdash A \sim_{\mathrm{n}} B & \text { normalized types } A \text { and } B \text { are equal }
\end{array}
$$

The first one is the general comparison of arguments $e$ and $e^{\prime}$ of an object boundary $\mathcal{B}$, the second one the type-directed phase which applies extensionality rules by matching the type, and the third the normalization phase which compares normalized expressions. We review the inductive clauses specifying these, shown in Figure 10. They are parametrized by a standard type theory $T$, a family of extensionality rules $\mathcal{E}$ over $T$, a family of computation rules $C$ over $T$, and a specification of principal arguments $\wp$. We again treat metavariables as primitive symbols.

General checking $\Theta ; \Gamma \vdash \mathscr{B} e \sim e^{\prime}$ descends under abstractions. It compares types by normalizing them, as there are no extensionality rules for types. Terms are compared by the type-directed phase, where the type is first normalized so that it can be matched against extensionality rules.

The type-directed phase checks $\Theta ; \Gamma \vdash u \sim_{\mathrm{e}} v: A$ by looking for an extensionality rule that matches $A$, and applying the rule to reduce the task to verification of the equational premises of the rule. The clause uses the notation $\mathscr{B} e \equiv e^{\prime}$ by $\square$, which turns an object 


$$
\begin{aligned}
& \frac{\Theta ; \Gamma \vdash\left(A \triangleright A^{\prime}\right) \text { type } \quad \Theta ; \Gamma \vdash u \sim_{\mathrm{e}} v: A^{\prime}}{\Theta ; \Gamma \vdash u \sim v: A} \\
& \frac{\Theta ; \Gamma \vdash\left(A \triangleright A^{\prime}\right) \text { type } \quad \Theta ; \Gamma \vdash\left(B \triangleright B^{\prime}\right) \text { type } \quad \Theta ; \Gamma \vdash\left(A^{\prime} \sim_{\mathrm{n}} B^{\prime}\right) \text { type }}{\Theta ; \Gamma \vdash(A \sim B) \text { type }} \\
& \frac{\mathrm{a} \notin|\Gamma| \quad \Theta ; \Gamma, \mathrm{a}: A \vdash(\mathscr{B}[\mathrm{a} / x]) e[\mathrm{a} / x] \sim e^{\prime}[\mathrm{a} / x]}{\Theta ; \Gamma \vdash\{x: A\} \mathscr{B}\left[\{x\} e \sim\{x\} e^{\prime}\right]} \\
& (\Xi, \mathrm{s}:(\square: P), \mathrm{t}:(\square: P), \Phi \Longrightarrow \mathrm{s} \equiv \mathrm{t}: P) \in \mathcal{E} \quad \Xi \vdash P \triangleright A \rightsquigarrow I \\
& \Theta ; \Gamma \vdash I_{*}\left(\mathscr{B} e \sim e^{\prime}\right) \quad \text { for }\left(\mathrm{M}: \mathscr{B} e \equiv e^{\prime} \text { by } \square\right) \in \Xi \\
& \frac{\Theta ; \Gamma \vdash\langle I, \mathrm{~s} \mapsto u, \mathrm{t} \mapsto v\rangle_{*}\left(\mathscr{B} e \sim e^{\prime}\right) \quad \text { for }\left(\mathrm{M}: \mathscr{B} e \equiv e^{\prime} \text { by } \square\right) \in \Phi}{\Theta ; \Gamma \vdash u \sim_{\mathrm{e}} v: A} \\
& \Xi \vdash P \triangleright A \not \psi \quad \text { for }(\Xi, \mathrm{s}:(\square: P), \mathrm{t}:(\square: P), \Phi \Longrightarrow \mathrm{s} \equiv \mathrm{t}: P) \in \mathcal{E} \\
& \Theta ; \Gamma \vdash u \triangleright u^{\prime}: A \quad \Theta ; \Gamma \vdash v \triangleright v^{\prime}: A \quad \Theta ; \Gamma \vdash u^{\prime} \sim_{\mathrm{n}} v^{\prime}: A \\
& \Theta ; \Gamma \vdash u \sim_{\mathrm{e}} v: A \\
& \overline{\Theta ; \Gamma \vdash \mathrm{a} \sim_{\mathrm{n}} \mathrm{a}: A}
\end{aligned}
$$

$$
\begin{aligned}
& \text { Rule for } \mathrm{S} \text { is } \mathrm{M}_{1}: \mathscr{B}_{1}, \ldots, \mathrm{M}_{n}: \mathscr{B}_{n} \Longrightarrow \boldsymbol{b}^{\prime} \mathrm{S}\left(\widehat{\mathrm{M}}_{1}, \ldots, \widehat{\mathrm{M}}_{n}\right) \\
& \Theta ; \Gamma \vdash\left(\left\langle\mathrm{M}_{1} \mapsto e_{1}, \ldots, \mathrm{M}_{i-1} \mapsto e_{i-1}\right\rangle_{*} \mathscr{B}_{i}\right) e_{i} \sim_{\mathrm{n}} e_{i}^{\prime} \quad \text { if } i \in \wp(\mathrm{S})
\end{aligned}
$$

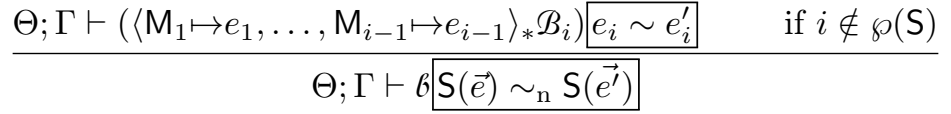

Figure 10: Equality checking with extensionality rules $\mathcal{E}$ and principal arguments $\wp$.

boundary into an equation boundary, as follows:

$$
\begin{aligned}
(\square: A) s \equiv t \text { by } \square & =(s \equiv t: A \text { by } \square), \\
(\square \text { type }) A \equiv B \text { by } \square & =(A \equiv B \text { by } \square), \\
(\{x: A\} \mathscr{B})\{x\} e \equiv\{x\} e^{\prime} \text { by } \square & =\{x: A\}\left(\mathscr{B} e \equiv e^{\prime} \text { by } \square\right) .
\end{aligned}
$$

If no extensionality rule applies, the terms $u$ and $v$ are normalized and compared by the normalization phase.

The normalization phase compares normalized expressions $\mathbf{S}(\vec{e})$ and $\mathbf{S}(\vec{e})$ by comparing their arguments, where the principal arguments are compared by the normalization phase because they have already been normalized, while the non-principal ones are subjected to general equality comparison.

The clauses in Figure 10 are readily turned into an equality-checking algorithm, because they are directed by the syntax of their goals. Application of extensionality rules is a possible source of non-determinism, as a type may match several extensionality rules, and also a source of non-termination, as there is no guarantee that eventually no extensionality rules will be applicable. We discuss strategies for dealing with these issues in Section 4.2. 
3.5. Soundness of equality checking. In this section we prove that the normalization and equality checking algorithms are sound. Because normalization and equality checking are intertwined, we prove Theorem 3.26 and Theorem 3.27 by mutual structural induction.

Theorem 3.26 (Soundness of normalization). In a standard type theory, given a family $C$ of computation rules, and a specification of principal arguments $\wp$, the following hold, where $\mathcal{B}$ and $\boldsymbol{b}$ are object boundaries:

(1) If $\Theta ; \Gamma \vdash \mathscr{B} e$ and $\Theta ; \Gamma \vdash \mathscr{B} e e^{\prime}$ then $\Theta ; \Gamma \vdash \mathscr{B} e \equiv e^{\prime}$ and $\Theta ; \Gamma \vdash \mathscr{B} e^{\prime}$.

(2) If $\Theta ; \Gamma \vdash b$ e and $\Theta ; \Gamma \vdash b \overline{e \triangleright_{\mathrm{p}} e^{\prime}}$ then $\Theta ; \Gamma \vdash b \overline{e \equiv e^{\prime}}$ and $\Theta ; \Gamma \vdash b \vec{e}$.

(3) If $\Theta ; \Gamma \vdash b e$ and $\Theta ; \Gamma \vdash b \overline{e \triangleright_{c} e^{\prime}}$ then $\Theta ; \Gamma \vdash b e \equiv e^{\prime}$ and $\Theta ; \Gamma \vdash b \overline{e^{\prime}}$.

Proof. We establish soundness of the rules from Figure 9 by mutual structural induction on the derivations. Derivability of $\Theta ; \Gamma \vdash \mathscr{B} e^{\prime}$ in (1) and of $\Theta ; \Gamma \vdash \mathscr{b} e^{\prime}$ in (2) and (3) follows already from Theorem 2.19, but we include these nonetheless as they will be needed in Theorem 3.27.

Part (1): The case of free variables follows by reflexivity and the variable rule.

If the derivation ends with

$$
\frac{\mathrm{a} \notin|\Gamma| \quad \Theta ; \Gamma, \mathrm{a}: A \vdash(\mathscr{B}[\mathrm{a} / x]) e[\mathrm{a} / x] \triangleright e^{\prime}}{\Theta ; \Gamma \vdash\{x: A\} \mathscr{B}[x\} e \triangleright\{x\} e^{\prime}[x / \mathrm{a}]}
$$

then by induction hypothesis

$$
\begin{aligned}
& \Theta ; \Gamma, \mathrm{a}: A \vdash(\mathcal{B}[\mathrm{a} / x]) e^{\prime}, \\
& \Theta ; \Gamma, \mathrm{a}: A \vdash(\mathcal{B}[\mathrm{a} / x]) e[\mathrm{a} / x] \equiv e^{\prime} .
\end{aligned}
$$

We may apply TT-ABstr to these, because $\Theta ; \Gamma \vdash A$ type holds by inversion on the assumption $\Theta ; \Gamma \vdash\{x: A\} \mathscr{B}\{x\} e$.

If the derivation ends with

$$
\begin{gathered}
\Theta ; \Gamma \vdash b\left[\begin{array}{l}
\mathrm{S}(\vec{e}) \triangleright_{\mathrm{p}} \mathrm{S}\left(\vec{e}^{\prime}\right) \\
\Xi \vdash p \triangleright \mathrm{S}\left(\vec{e}^{\prime}\right) \not \leftrightarrow \quad \text { for }\left(\Xi \Longrightarrow \mathfrak{b}^{\prime} p \equiv v\right) \in C
\end{array}\right. \\
\Theta ; \Gamma \vdash b\left[\mathrm{~S}(\vec{e}) \triangleright \mathrm{S}\left(\vec{e}^{\prime}\right)\right.
\end{gathered}
$$

then the claim follows by the induction hypothesis (2) for the first premise.

The remaining case is

$$
\frac{\Theta ; \Gamma \vdash b \longdiv { \mathrm { S } ( \vec { e } ) \triangleright _ { \mathrm { p } } \mathrm { S } ( \vec { e } ^ { \prime } ) } \quad \Theta ; \Gamma \vdash b \overrightarrow{\mathrm{S}\left(\overrightarrow{e^{\prime}}\right) \triangleright_{\mathrm{c}} e^{\prime \prime}} \quad \Theta ; \Gamma \vdash b \overrightarrow{e^{\prime \prime} \triangleright e^{\prime \prime \prime}}}{\Theta ; \Gamma \vdash b \overline{\mathrm{S}(\vec{e}) \triangleright e^{\prime \prime \prime}}}
$$

The induction hypothesis for the last premise secures $\Theta ; \Gamma \vdash b e^{\prime \prime \prime}$, while the induction hypotheses for all three premises yield

$$
\Theta ; \Gamma \vdash b \mathrm{~S}(\vec{e}) \equiv \mathrm{S}\left(\overrightarrow{e^{\prime}}\right), \quad \Theta ; \Gamma \vdash b \overrightarrow{\mathrm{S}\left(\overrightarrow{e^{\prime}}\right) \equiv e^{\prime \prime},}, \quad \Theta ; \Gamma \vdash b e^{\prime \prime} \equiv e^{\prime \prime \prime} .
$$

We may string these together using transitivity to derive $\Theta ; \Gamma \vdash b \mathrm{~S}(\vec{e}) \equiv e^{\prime \prime \prime}$.

Part (2): Suppose the rule for $\mathrm{S}$ is

$$
\mathrm{M}_{1}: \mathscr{B}_{1}, \ldots, \mathrm{M}_{n}: \mathscr{B}_{n} \Longrightarrow \mathfrak{b}^{\prime} \mathrm{S}\left(\widehat{\mathrm{M}}_{1}, \ldots, \widehat{\mathrm{M}}_{n}\right)
$$


and consider normalization of principal arguments

$$
\begin{array}{r}
\Theta ; \Gamma \vdash\left(I_{(i) *} \mathcal{B}_{i}\right) e_{i} \triangleright e_{i}^{\prime} \quad \text { if } i \in \wp(\mathrm{S}) \\
e_{i}=e_{i}^{\prime} \quad \text { if } i \notin \wp(\mathrm{S}) \\
\Theta ; \Gamma \vdash b \mathrm{~S}(\vec{e}) \triangleright_{\mathrm{p}} \mathrm{S}\left(\vec{e}^{\prime}\right)
\end{array}
$$

where $I=\left\langle\mathrm{M}_{1} \mapsto e_{1}, \ldots, \mathrm{M}_{n} \mapsto e_{n}\right\rangle$. For $i=1, \ldots, n$, we have

$$
\Theta ; \Gamma \vdash\left(I_{(i) *} \mathscr{B}_{i}\right) e_{i} \equiv e_{i}^{\prime} \quad \text { and } \quad \Theta ; \Gamma \vdash\left(I_{(i) *} \mathscr{B}_{i}\right) e_{i} \text {. }
$$

Indeed, for $i \in \wp(\mathrm{S})$ the above are just the induction hypotheses of a premise, while for $i \notin \wp(\mathrm{S})$ they respectively hold by reflexivity and an application of Corollary 2.24 to $\Theta ; \Gamma \vdash b \overrightarrow{\mathrm{S}(\vec{e})}$. Therefore, the instantiation $J=\left\langle\mathrm{M}_{1} \mapsto e_{1}^{\prime}, \ldots, \mathrm{M}_{n} \mapsto e_{n}^{\prime}\right\rangle$ is judgementally equal to $I$, and because $I$ is derivable, $J$ is derivable by Proposition 2.28. From these facts we conclude

$$
\begin{array}{ll}
\Theta ; \Gamma \vdash\left(I_{*} b^{\prime}\right) \mathrm{S}(\vec{e}) \equiv \mathrm{S}\left(\vec{e}^{\prime}\right) & \text { by the congruence rule for } S, \\
\Theta ; \Gamma \vdash\left(J_{*} b^{\prime}\right) \mathrm{S}\left(\vec{e}^{\prime}\right) & \text { by the rule for } S .
\end{array}
$$

If $\mathfrak{b}^{\prime}=\left(\square\right.$ type), we are done. If $\mathfrak{b}^{\prime}=(\square: A)$ and $\mathfrak{b}=(\square: B)$ then we derive $\Theta ; \Gamma \vdash I_{*} A \equiv$ $J_{*} A$ by Theorem 2.18 and $\Theta ; \Gamma \vdash I_{*} A \equiv B$ by Theorem 2.22 on $\Theta ; \Gamma \vdash b[\mathrm{~S}(\vec{e})$ and convert the judgements along them.

Part (3): Consider an application of a type computation rule

$$
\begin{gathered}
(\Xi \Longrightarrow P \equiv B) \in C \quad \Xi \vdash P \triangleright A \rightsquigarrow I \\
\frac{\Theta ; \Gamma \vdash I_{*}\left(\mathcal{B} e \sim e^{\prime}\right) \quad \text { for }\left(\mathrm{M}: \mathcal{B} e \equiv e^{\prime} \text { by } \square\right) \in \Xi}{\Theta ; \Gamma \vdash A \triangleright_{\mathrm{c}} I_{*} B}
\end{gathered}
$$

Theorem 3.27 ensures $\Theta ; \Gamma \vdash I_{*}\left(\mathscr{B} \overline{e \equiv e^{\prime}}\right)$ for every $\left(\mathrm{M}: \mathscr{B} e \equiv e^{\prime}\right.$ by $\left.\square\right) \in \Xi$. Therefore, since $\Xi \Longrightarrow P$ type is object-invertible and $\Theta ; \Gamma \vdash I_{*} P$ type has been assumed (note that $I_{*} P=A$ ), it follows by Proposition 3.10 that $I$ is derivable. We now instantiate the computation rule $\Xi \Longrightarrow P \equiv B$ by $I$ to get $\Theta ; \Gamma \vdash A \equiv I_{*} B$ and appeal to Theorem 2.19 for $\Theta ; \Gamma \vdash I_{*} B$ type.

It remains to establish the soundness of a derivation ending with a term computation rule

$$
\begin{gathered}
(\Xi \Longrightarrow p \equiv v: B) \in C \quad \Xi \vdash p \triangleright s \rightsquigarrow I \\
\frac{\Theta ; \Gamma \vdash I_{*}\left(\mathcal{B} e \sim e^{\prime}\right) \quad \text { for }\left(\mathrm{M}: \mathcal{B} e \equiv e^{\prime} \text { by } \square\right) \in \Xi}{\Theta ; \Gamma \vdash s \triangleright_{\mathrm{c}} I_{*} v: A}
\end{gathered}
$$

Theorem 3.27 ensures $\Theta ; \Gamma \vdash I_{*}\left(\mathcal{B} e \equiv e^{\prime}\right)$ for every $\left(\mathrm{M}: \mathcal{B} e \equiv e^{\prime}\right.$ by $\left.\square\right) \in \Xi$. Observe that since by Definition $3.19 p$ is a term symbol application, $\operatorname{mv}\left(\tau_{\Xi ;[]}(p)\right) \subseteq \operatorname{mv}(p)$ and $I_{*} p=s$ imply $I_{*}\left(\tau_{\Xi ;[]}(p)\right)=\tau_{\Theta ; \Gamma}(s)$ by Proposition 2.23. Because $\Theta ; \Gamma \vdash s: A$ is derivable, so is $\Theta ; \Gamma \vdash s: \tau_{\Theta ; \Gamma}(s)$ by Corollary 2.25, which equals $\Theta ; \Gamma \vdash I_{*} p: I_{*}\left(\tau_{\Theta ; \Gamma}(p)\right)$. We may apply Proposition 3.10 to the object-invertible rule $\Xi \Longrightarrow p: \tau_{\Theta ; \Gamma}(p)$ to establish that $I$ is derivable. By instantiating the computation rule $\Xi \Longrightarrow p \equiv v: B$ with $I$ we obtain

$$
\Theta ; \Gamma \vdash s \equiv I_{*} v: I_{*} B
$$

and convert it along $\Theta ; \Gamma \vdash I_{*} B \equiv A$ to the desired form, because Theorem 2.19 implies $\Theta ; \Gamma \vdash s: I_{*} B$ and Theorem 2.22 that $\Theta ; \Gamma \vdash I_{*} B \equiv A$. The last claim follows once again from Theorem 2.19. 
Theorem 3.27 (Soundness of equality checking). In a standard type theory, given families $C$ and $\mathcal{E}$ of computation and extensionality rules, and a specification of principal arguments $\wp$, the following hold, where $\mathcal{B}$ is an object boundary:

(1) $\Theta ; \Gamma \vdash \mathscr{B} e \equiv e^{\prime}$ holds if

$$
\Theta ; \Gamma \vdash \mathscr{B} e, \quad \Theta ; \Gamma \vdash \mathscr{B} e^{\prime}, \quad \text { and } \quad \Theta ; \Gamma \vdash \mathscr{B} e \sim e^{\prime} .
$$

(2) $\Theta ; \Gamma \vdash u \equiv v: A$ holds if

$$
\Theta ; \Gamma \vdash u: A, \quad \Theta ; \Gamma \vdash v: A, \quad \text { and } \quad \Theta ; \Gamma \vdash u \sim_{\mathrm{e}} v: A .
$$

(3) $\Theta ; \Gamma \vdash A \equiv B$ holds if

$$
\Theta ; \Gamma \vdash A \text { type, } \quad \Theta ; \Gamma \vdash B \text { type, and } \Theta ; \Gamma \vdash A \sim_{\mathrm{n}} B .
$$

(4) $\Theta ; \Gamma \vdash u \equiv v: A$ holds if

$$
\Theta ; \Gamma \vdash u: A, \quad \Theta ; \Gamma \vdash v: A, \quad \text { and } \quad \Theta ; \Gamma \vdash u \sim_{\mathrm{n}} v: A .
$$

Proof. We proceed by mutual structural induction on the derivation.

Part (1): Consider a derivation ending with an abstraction

$$
\begin{aligned}
& \mathrm{a} \notin|\Gamma| \quad \Theta ; \Gamma, \mathrm{a}: A \vdash(\mathscr{B}[\mathrm{a} / x]) e[\mathrm{a} / x] \sim e^{\prime}[\mathrm{a} / x] \\
& \Theta ; \Gamma \vdash\{x: A\} \mathscr{B}\{x\} e \sim\{x\} e^{\prime}
\end{aligned}
$$

By inverting the assumptions we get

$$
\Theta ; \Gamma, \mathrm{a}: A \vdash \mathcal{B}[\mathrm{a} / x] e[\mathrm{a} / x] \quad \text { and } \quad \Theta ; \Gamma, \mathrm{a}: A \vdash \mathscr{B}[\mathrm{a} / x] e^{\prime}[\mathrm{a} / x],
$$

as well as $\Theta ; \Gamma \vdash\{x: A\} \mathscr{B}\{x\} e$. Now the induction hypothesis for the premise yields

$$
\Theta ; \Gamma, \mathrm{a}: A \vdash(B[\mathrm{a} / x]) e[\mathrm{a} / x] \equiv e^{\prime}[\mathrm{a} / x],
$$

which we may abstract with TT-ABSTR.

If the derivation ends with

$$
\frac{\Theta ; \Gamma \vdash\left(A \triangleright A^{\prime}\right) \text { type } \quad \Theta ; \Gamma \vdash\left(B \triangleright B^{\prime}\right) \text { type } \quad \Theta ; \Gamma \vdash\left(A^{\prime} \sim_{\mathrm{n}} B^{\prime}\right) \text { type }}{\Theta ; \Gamma \vdash(A \sim B) \text { type }}
$$

then Theorem 3.26 applied to the first two premises gives

$$
\begin{array}{ll}
\Theta ; \Gamma \vdash A \equiv A^{\prime}, & \Theta ; \Gamma \vdash A^{\prime} \text { type, } \\
\Theta ; \Gamma \vdash B \equiv B^{\prime}, & \Theta ; \Gamma \vdash B^{\prime} \text { type, }
\end{array}
$$

and then the induction hypothesis for the last premise $\Theta ; \Gamma \vdash A^{\prime} \equiv B^{\prime}$. From these we may derive $\Theta ; \Gamma \vdash A \equiv B$ easily.

Suppose the derivation ends with

$$
\frac{\Theta ; \Gamma \vdash\left(A \triangleright A^{\prime}\right) \text { type } \quad \Theta ; \Gamma \vdash u \sim_{\mathrm{e}} v: A^{\prime}}{\Theta ; \Gamma \vdash u \sim v: A}
$$

By Theorem 2.19 applied to the assumption we see that $\Theta ; \Gamma \vdash A$ type, hence we may apply Theorem 3.26 to the first premise and get

$$
\Theta ; \Gamma \vdash A \equiv A^{\prime} \quad \text { and } \quad \Theta ; \Gamma \vdash A^{\prime} \text { type }
$$

We convert the assumptions along the above equation to

$$
\Theta ; \Gamma \vdash u: A^{\prime} \quad \text { and } \quad \Theta ; \Gamma \vdash v: A^{\prime}
$$


so that we may apply the induction hypothesis to the second premise and obtain $\Theta ; \Gamma \vdash u \equiv$ $v: A^{\prime}$. One more conversion is then needed to derive $\Theta ; \Gamma \vdash u \equiv v: A$.

Part (2): If the derivation ends with

$$
\begin{aligned}
& \Xi \vdash A \triangleright P \not \rightarrow \quad \text { for }(\Xi, \mathrm{s}:(\square: P), \mathrm{t}:(\square: P), \Phi \Longrightarrow \mathrm{s} \equiv \mathrm{t}: P) \in \mathcal{E} \\
& \begin{array}{ccc}
\Theta ; \Gamma \vdash u \triangleright u^{\prime}: A \quad \Theta ; \Gamma \vdash v \triangleright v^{\prime}: A \quad \Theta ; \Gamma \vdash u^{\prime} \sim_{\mathrm{n}} v^{\prime}: A \\
\Theta ; \Gamma \vdash u \sim_{\mathrm{e}} v: A
\end{array}
\end{aligned}
$$

then Theorem 3.26 applied to the first two premises establishes

$$
\begin{array}{ll}
\Theta ; \Gamma \vdash u \equiv u^{\prime}: A & \Theta ; \Gamma \vdash u^{\prime}: A \\
\Theta ; \Gamma \vdash v \equiv v^{\prime}: A & \Theta ; \Gamma \vdash v^{\prime}: A
\end{array}
$$

Then the induction hypothesis tells us that $\Theta ; \Gamma \vdash u^{\prime} \equiv v^{\prime}: A$. It is now easy to combine the derived equalities into $\Theta ; \Gamma \vdash u \equiv v: A$.

If the derivation ends with an application of an extensionality rule

$$
\begin{array}{cc}
(\Xi, \mathrm{s}:(\square: P), \mathrm{t}:(\square: P), \Phi \Longrightarrow \mathrm{s} \equiv \mathrm{t}: P) \in \mathcal{E} \quad \Xi \vdash A \triangleright P \rightsquigarrow I \\
\Theta ; \Gamma \vdash I_{*}\left(\mathbb{B} e \sim e^{\prime}\right) & \text { for }\left(\mathrm{M}: \mathscr{B} e \equiv e^{\prime} \text { by } \square\right) \in \Xi \\
\Theta ; \Gamma \vdash\langle I, \mathrm{~s} \mapsto u, \mathrm{t} \mapsto v\rangle_{*}\left(\mathscr{B} e \sim e^{\prime}\right) & \text { for }\left(\mathrm{M}: \mathscr{B} e \equiv e^{\prime} \text { by } \square\right) \in \Phi \\
\hline ; \Gamma \vdash u \sim \sim_{\mathrm{e}} v: A
\end{array}
$$

then $\Theta ; \Gamma \vdash A$ type follows from $\Theta ; \Gamma \vdash u: A$ by Theorem 2.19. Induction hypotheses for the premises give

$$
\begin{array}{ll}
\Theta ; \Gamma \vdash I_{*}\left(\mathscr{B} e \equiv e^{\prime}\right) & \text { for }\left(\mathrm{M}: \mathcal{B} e \equiv e^{\prime} \text { by } \square\right) \in \Xi \\
\Theta ; \Gamma \vdash\langle I, \mathrm{~s} \mapsto u, \mathrm{t} \mapsto v\rangle_{*}\left(\mathscr{B} e \equiv e^{\prime}\right) & \text { for }\left(\mathrm{M}: \mathcal{B} e \equiv e^{\prime} \text { by } \square\right) \in \Phi
\end{array}
$$

Because $\Xi \Longrightarrow P$ type is object-invertible, and $I_{*} P=A$ and $\Theta ; \Gamma \vdash A$ type is derivable, by Proposition 3.10 the instantiation $I$ is derivable too. We extend $I$ to the instantiation

$$
J=\langle I, \mathrm{~s} \mapsto u, \mathrm{t} \mapsto v, \Phi \mapsto \star\rangle
$$

of the premises of the extensionality rule over $\Theta ; \Gamma$, where $\Phi \mapsto \star$ signifies that the metavariables of $\Phi$ are instantiated with (suitably abstracted) dummy values. We claim that $J$ is derivable: we already know that $I$ is derivable; derivability at $\mathrm{s}$ and $\mathrm{t}$ reduces to the assumptions $\Theta ; \Gamma \vdash u: A$ and $\Theta ; \Gamma \vdash u: A$; and derivability at $\Phi$ holds by the induction hypotheses (3.13). When we instantiate the extensionality rule with $J$, we obtain the desired equation.

Parts (3) and (4): The variable case $\Theta ; \Gamma \vdash \mathrm{a} \sim_{\mathrm{n}} \mathrm{a}: A$ is trivial.

Suppose the rule for symbol $\mathrm{S}$ is

$$
\mathrm{M}_{1}: \mathscr{B}_{1}, \ldots, \mathrm{M}_{n}: \mathscr{B}_{n} \Longrightarrow \boldsymbol{b}^{\prime} \mathrm{S}\left(\widehat{\mathrm{M}}_{1}, \ldots, \widehat{\mathrm{M}}_{n}\right)
$$

and the derivation ends with

$$
\begin{aligned}
& \Theta ; \Gamma \vdash\left(I_{(i) *} \mathscr{B}_{i}\right) e_{i} \sim_{\mathrm{n}} e_{i}^{\prime} \quad \text { if } i \in \wp(\mathrm{S}) \\
& \underline{\Theta ; \Gamma \vdash\left(I_{(i) *} \mathscr{B}_{i}\right) e_{i} \sim e_{i}^{\prime} \quad \text { if } i \notin \wp(\mathrm{S})}
\end{aligned}
$$

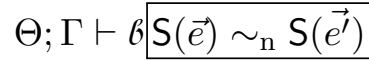

where $I=\left\langle\mathrm{M}_{1} \mapsto e_{1}, \ldots, \mathrm{M}_{n} \mapsto e_{n}\right\rangle$, and define $J=\left\langle\mathrm{M}_{1} \mapsto e_{1}^{\prime}, \ldots, \mathrm{M}_{n} \mapsto e_{n}^{\prime}\right\rangle$. We first derive

$$
\Theta ; \Gamma \vdash\left(I_{*} b^{\prime}\right) \mathrm{S}(\vec{e}) \equiv \mathrm{S}\left(\overrightarrow{e^{\prime}}\right)
$$


by the congruence rule associated with $\mathrm{S}$, whose premises are derived as follows:

(1) For each $i=1, \ldots, n$ the premise $\Theta ; \Gamma \vdash\left(I_{(i) *} \mathscr{B}_{i}\right) e_{i}$ is derivable by Corollary 2.24 applied to $\Theta ; \Gamma \vdash \mathfrak{b} \mathrm{S}(\vec{e})$. This also shows that $I$ is derivable.

(2) For each $i=1, \ldots, n$ such that $\mathscr{B}_{i}$ is an object boundary, the premise $\Theta ; \Gamma \vdash\left(I_{(i) *} \mathscr{B}_{i}\right) e_{i} \equiv e_{i}^{\prime}$ is one of the induction hypotheses. This also shows that $I$ and $J$ are judgementally equal, therefore $J$ is derivable by Proposition 2.28.

(3) For each $i=1, \ldots, n$ the premise $\Theta ; \Gamma \vdash\left(J_{(i) *} \mathcal{B}_{i}\right) e_{i}^{\prime}$ is derivable because $J$ is derivable.

If $\mathfrak{b}=\square$ type, we are done. If $\mathfrak{b}^{\prime}=(\square: A)$ and $\mathfrak{b}=(\square: B)$, we convert (3.14) along $\Theta ; \Gamma \vdash I_{*} A \equiv B$. The equation holds by Theorem 2.22 applied to $\Theta ; \Gamma \vdash \mathrm{S}(\vec{e}): B$ and $\Theta ; \Gamma \vdash \mathrm{S}(\vec{e}): I_{*} A$, where the latter is derived by Theorem 2.19 and the former by the rule for $S$.

\section{Discussion}

The relations defined by the inductive clauses from Figures 9 and 10 serve as the basis of an equality checking algorithm. In order to obtain a working and useful implementation, we need to address several issues.

4.1. Classification of rules and principal arguments. An experienced designer of type theories is quite able to recognize computation and extensionality rules, and stitch them together by picking correct principal arguments. There is no need for such manual work, because Propositions 3.20 and 3.23 provide easily verifiable syntactic criteria for recognizing computation and extensionality rules. The principal arguments must be chosen correctly, lest the equality checking procedure fail unnecessarily or enter an infinite loop, as shown by the following example.

Example 4.1. Consider the computation and extensionality rules for simple products shown in Figure 11, where we ignore the linearity requirements, as they just obscure the point we wish to make. Without any principal arguments, the algorithm fails to apply the first

$$
\begin{aligned}
& \frac{\vdash \text { A type } \quad \vdash \text { B type } \quad \vdash \mathrm{s}: \mathrm{A} \quad \vdash \mathrm{t}: \mathrm{B}}{\vdash \text { fst }(\mathrm{A}, \mathrm{B}, \operatorname{pair}(\mathrm{A}, \mathrm{B}, \mathrm{s}, \mathrm{t})) \equiv \mathrm{s}: \mathrm{A}} \\
& \frac{\vdash \text { A type } \vdash \text { B type } \vdash \mathrm{s}: \mathrm{A} \quad \vdash \mathrm{t}: \mathrm{B}}{\vdash \operatorname{snd}(\mathrm{A}, \mathrm{B}, \operatorname{pair}(\mathrm{A}, \mathrm{B}, \mathrm{s}, \mathrm{t})) \equiv \mathrm{t}: \mathrm{B}} \\
& \vdash \text { A type } \quad \vdash \text { B type } \quad \vdash \mathrm{s}: \mathrm{A} \times \mathrm{B} \\
& \frac{\vdash \mathrm{t}: \mathrm{A} \times \mathrm{B} \quad \vdash \mathrm{fst}(\mathrm{A}, \mathrm{B}, \mathrm{s}) \equiv \mathrm{fst}(\mathrm{A}, \mathrm{B}, \mathrm{t}): \mathrm{A} \quad \vdash \operatorname{snd}(\mathrm{A}, \mathrm{B}, \mathrm{s}) \equiv \operatorname{snd}(\mathrm{A}, \mathrm{B}, \mathrm{t}): \mathrm{B}}{\vdash \mathrm{s} \equiv \mathrm{t}: \mathrm{A} \times \mathrm{B}}
\end{aligned}
$$

Figure 11: Computation and extensionality rules for simple products

computation rule to fst $(A, B, u)$ in case $u$ normalizes to a pair. More ominous is the infinite loop that is entered on checking

$$
\text { []; } x: A \times B, y: A \times B \vdash x \equiv y: A \times B,
$$


where we assume that $A$ and $B$ are already normalized. The algorithm performs the following steps (where all judgements are placed in the variable context []; $x: A \times B, y: A \times B$ ). First, the extensionality phase reduces the equation to

$$
\mathrm{fst}(A, B, x) \equiv \mathrm{fst}(A, B, y): A, \quad \operatorname{snd}(A, B, x) \equiv \operatorname{snd}(A, B, y): B .
$$

after which the normalization verifies the first equation by comparing

$$
A \equiv A, \quad B \equiv B, \quad x \equiv y: A \times B .
$$

We may short-circuit the first two equalities, but checking the third one leads back to the original one, unless the third argument of fst is principal, in which case the algorithm persists in the normalization phase and fails immediately, as it should.

The previous example suggests that we can read off the principal arguments either from extensionality rules, by looking for occurrences of the left and right-hand sides in the subsidiary equalities, or from computation rules, by inspecting the syntactic form of the left-hand side of the rule. We have analyzed a number of standard computation and extensionality rules and identified the following strategy for automatic determination of principal arguments, which we also implemented:

The $i$-th argument of $\mathbf{S}$ is principal if there is a computation rule $\Xi \Longrightarrow$ $b p \equiv v$ such that $\mathrm{S}\left(e_{1}, \ldots, e_{n}\right)$ appears as a sub-pattern of $p$ and $e_{i}$ is neither of the form $\mathrm{M}()$ nor $\{\vec{x}\} \mathrm{M}(\vec{x})$.

In many cases, among others the simply-typed $\lambda$-calculus, inductive types, and intensional Martin-Löf type theory, the strategy leads to weak head-normal forms. We postpone the pursuit of deeper understanding of this phenomenon to another time.

It would be interesting to combine principal arguments with another common technique for controlling applications of extensionality rules, namely neutral forms. Roughly, the principal arguments would still tell which arguments are normalized, but not whether they are compared structurally. Instead, we always compare them recursively, but skip the type-directed phase in $s \equiv t: A$ when the syntactic forms of $s$ and $t$ are neutral, i.e., they indicate that application of extensionality rules cannot lead to further progress. For instance, when checking $x \equiv y: A \times B$ in Example 4.1, there is no benefit to applying projections to the variables $x$ and $y$. Each specific type theory has its own neutral forms, if any, so the user would have to describe these. In some cases it might even be possible to detect the neutral forms automatically.

4.2. Determinism, termination and completeness. The inductive clauses in Figures 9 and 10 could be implemented either as proof search, or as a streamlined algorithm based on normalization. Proof assistants typically implement the latter strategy, because they work with type theories whose normalization is confluent and terminating, and equality checking requires no backtracking. We use the same strategy, so we ought to address non-determinism and non-termination.

A computation or extensionality rule cannot be the source of non-determinism on its own, because Definitions 3.18, 3.19 and 3.22 prescribe determinism. However, in either phase of the algorithm several rules may be applicable at the same time, which leads to non-determinism, and we saw in Example 4.1 that a poor choice of principal arguments causes non-termination. This is all quite familiar, and so are techniques for ensuring that all is well, including confluence checking and termination arguments based on well-founded relations. 
While these are doubtlessly important issues, we are not addressing them because they are independent of the algorithm itself. Instead, we aim to provide equality checking that favors generality and extensibility, while still providing soundness through Theorems 3.26 and 3.27. In this regard we are in good company, as recent version of Agda allow potentially unsafe user-defined computation rules, a point further discussed in Section 7.

A related question is completeness of equality checking, i.e., does the algorithm succeed in checking every derivable equation? Once again, our position is the same: completeness is important, both theoretically and from a practical point of view, but is not the topic of the present paper. Numerous techniques for establishing completeness of equality checking are known, and these can be applied to any specific instantiation of our algorithm. An interesting direction to pursue would be adaptation of such techniques to our general setting.

\section{IMPLEMENTATION}

Having laid out the algorithm, we report on our experience with its implementation in the Andromeda 2 proof assistant $\left[\mathrm{BGH}^{+} 18\right.$, And, BHP20], in which the user may define any work in any standard type theory. It is an LCF style proof assistant, i.e., a meta-level programming language with abstract datatypes of judgements, boundaries, and derived rules whose construction and application is controlled by a trusted nucleus (consisting of around 4200 lines of OCaml code).

The nucleus implements context-free type theory, a variant of type theory in which there are no contexts. Instead, each free variable is tagged with its type and each metavariable with its boundary, as explained in [HB21]. Since there are no contexts, a mechanism is needed for tracking proof-irrelevant uses of metavariables and variables, which may occur in derivations of equalities. For this purpose, equality judgements take the form

$$
A \equiv B \text { by } \alpha \quad \text { and } \quad s \equiv t: A \text { by } \alpha
$$

where $\alpha$ is an assumption set whose elements are those metavariables and variables that are used to derive the equality but do not appear in its boundary. The assumptions sets are also recorded in term conversions. As far as the equality checking algorithm is concerned, this is an annoying but inessential complication, because all conversions must be performed explicitly and carefully accounted for.

The implementation of the equality checking algorithm comprises around 1400 lines of OCaml code which reside outside of the trusted nucleus, so that each reasoning step must be passed to the nucleus for validation. The overhead of such a policy is significant, but worth paying in exchange for keeping the nucleus small and uncorrupted, at least in the initial, experimental phase.

Our rudimentary implementation is quite inefficient and cannot compete with the equality checkers found in mature proof assistants. The interesting question is not whether we could try harder to significantly speed up the algorithm, which presumably we could, but whether the design of the algorithm makes it inherently inefficient. We argue that this is not the case. First, we may trade safety for efficiency by placing equality checking into the trusted nucleus, as many proof assistants do, so that we need not check every single step of the algorithm. Second, even though term equality is typed, the normalization procedure is essentially untyped. Indeed, when the rules in Figure 9 are used to normalize $\Theta ; \Gamma \vdash t: A$ they never modify $A$, and only ever inspect $t$, which allows us to ignore $A$ while rewriting $t$. The soundness of the algorithm guarantees that the normalized term will still have type $A$. 


\section{EXAmples}

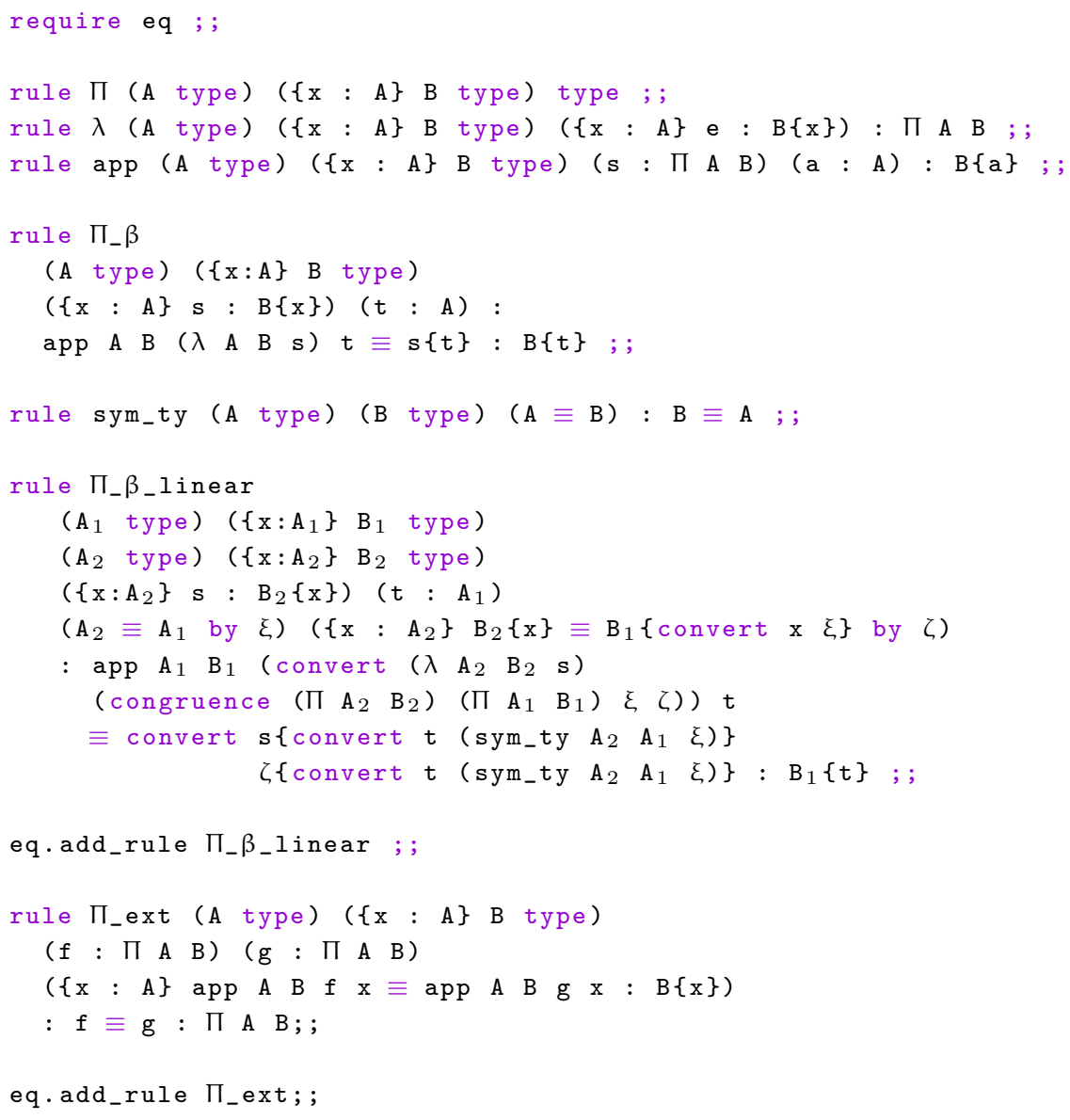

Figure 12: Dependent products in Andromeda 2.

The example in Figure 12 shows how dependent products are formalized in Andromeda 2. The rules are direct transcriptions of the usual ones. We linearize the $\beta$-rule as shown in Example 3.4 to make it a computation rule. We do so by explicitly converting $\lambda \mathrm{A}_{2} \mathrm{~B}_{2} \mathrm{~s}$ along the equality $\Pi A_{2} B_{2} \equiv \Pi A_{1} B_{1}$, which holds by a congruence rule and the premises $\xi$ and $\zeta$.

The calls to eq.add_rule pass equality rules to the equality checking algorithm, which employs Propositions 3.20 and 3.23 to automatically classify the inputs as computation or extensionality rules. It also determines which arguments are principal by using the technique from Section 4.1. In the example shown, the linearized rule $\Pi_{-} \beta_{-}$linear is classified by the algorithm as computation rule, $\Pi_{-}$ext as extensionality rule, and the the third argument of app is declared principal.

Many a newcomer to Martin-Löf type theory is disappointed to learn that only one of equalities $0+n=n$ and $n+0=n$ holds judgementally. In fact, there is strong temptation to pass to extensional type theory just so that a more symmetric notion of equality is recovered, but then one has to give up decidable equality checking. The example in Figures 13 
and 14 shows how our algorithm combines the best of both worlds and demonstrates further capabilities of the implementation.

First, Figure 13 shows a formalization of extensional equality types, whose distinguishing feature is the equality reflection principle equality_reflection, which states that the equality type Eq reflects into judgemental equality. Instead of postulating the familiar eliminator $\mathrm{J}$, it is more convenient to use an equivalent formulation that uses the judgemental uniqueness of equality proofs uip, see Example 3.25. Note that uip is installed as an extensionality rule into the equality checker. It is well known that equality reflection makes equality checking undecidable, so the equality checker will not be able to prove all equalities. Nevertheless, we expect it to be still quite useful and well behaved.

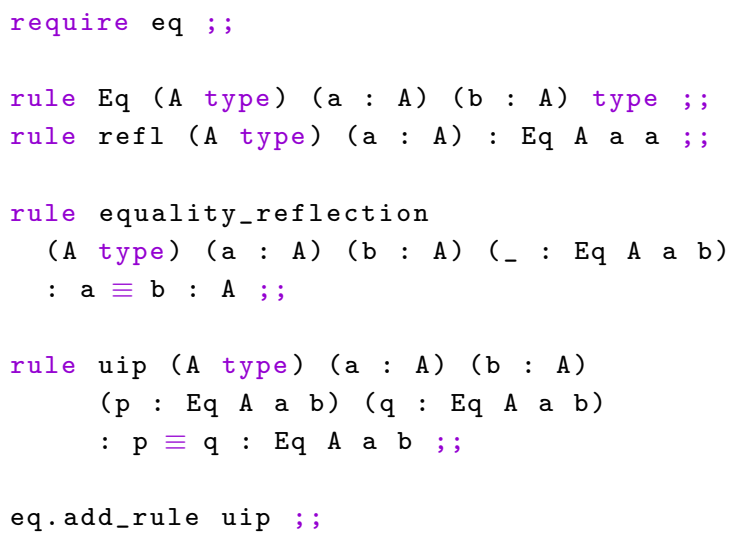

Figure 13: Extensional equality type in Andromeda 2.

We continue our example in Figure 14 by postulating the natural numbers $\mathbb{N}$. Everything up to the definition of addition is standard, where we also install the computation rules for the induction principle $\mathbb{N}_{-}$ind into the equality checker. We then define addition by postulating a term symbol + with the defining equality plus_def which expresses addition by primitive recursion. We could use plus_def as a global computation rule, but we choose to use it only locally, with the help of the function eq.add_locally.

In the remainder of the code we prove judgemental equalities

$$
n+0 \equiv n, \quad m+\operatorname{succ}(n) \equiv \operatorname{succ}(m+n), \quad \text { and } \quad 0+n=n .
$$

The first one is derived as plus_zero_right using plus_def as a local computation rule together with eq.prove which takes an equational boundary (where $\square$ is written as ??) and runs the equality checking algorithm to generate a witness for it. The second equality is derived as plus_succ in much the same way. The derivation of the third equality relies on equality reflection to convert a term of the equality type $\mathrm{Eq} \mathbb{N}$ (zero $+\mathrm{n}$ ) $\mathrm{n}$ to the corresponding judgemental equality zero $+\mathrm{n} \equiv \mathrm{n}: \mathbb{N}$. We install all three equalities as computation rules.

In addition to proving equalities, we can also normalize terms with eq.normalize, and compute strong normal forms (all arguments are principal) with eq.compute. In both cases we obtain not only the result, but also a certifying equality. For example, when given succ zero + succ zero, the normalizer outputs the weak head-normal form succ ((succ zero) + zero), together with a certificate for the judgemental equality (succ zero) + (succ zero) $\equiv$ succ ((succ zero) + zero) $: \mathbb{N}$. Because we installed both neutrality laws for 0 as computation rules, strong 


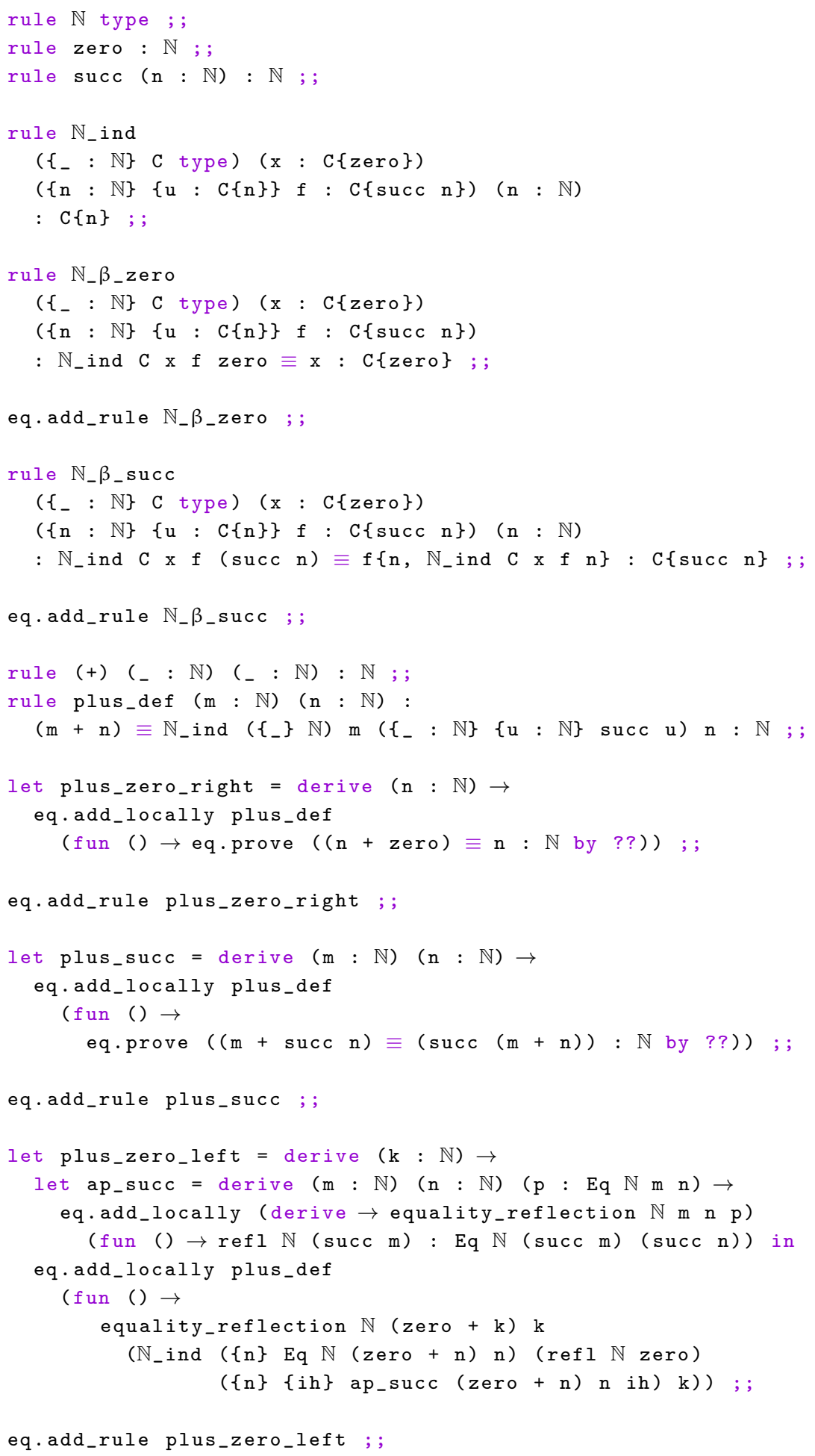

Figure 14: Addition for natural numbers in Andromeda 2. 
normalization reduces (zero $+\mathrm{x}$ ) $+\operatorname{succ}($ succ zero + zero) to succ ( $\operatorname{succ} \mathrm{x}$ ) : $\mathbb{N}$, where $\mathrm{x}$ is a free variable of type $\mathbb{N}$.

\section{RELATED WORK}

Designing a user-extensible equality checking algorithm for type theory is a balancing act between flexibility, safety, and automation. We compare ours to that of several proof assistants that support user-extensible equality checking.

The overall design of our algorithm is similar to the equality checking and simplification phases used in the type-reconstruction algorithm of MMT [MMT, Rab18], a meta-metalanguage for description of formal theories. In MMT inference rules are implemented as trusted low-level executable code, which gives the system an extremely wide scope but also requires care and expertise by the user. In Andromeda 2 the user writes down the desired inference rules directly. The nucleus checks them for compliance with Definition 2.13 of a standard type theory before accepting them, which prevents the user from breaking the meta-theoretic properties that the nucleus relies on.

Dedukti [Ded] is a type-checker founded on the logical framework $\lambda \Pi$, extended with user-defined conversion rules. Because equality in Dedukti is based on convertibility of terms, there is no support for user-defined extensionality or $\eta$-rules. The Dedukti rewriting system supports higher-order patterns and includes a confluence checker. We see no obstacle to adding some form of confluence checking to Andromeda 2 in the future, while support for higher-order patterns would first have to overcome lack of strengthening, see the discussion following Definition 3.2.

Recent versions of the proof assistant Agda support user-definable computation rules [CA16, Coc20, CTW21]. Like Dedukti, Agda allows higher-order patterns and provides a confluence checker. It accepts non-linear patterns, which it linearizes and generates suitable equational premises. In addition, it applies built-in $\eta$-rules for functions and record types during a typedirected matching phase. It seems to us that the phase could equally well use extensionality rules, which might more easily enable user-defined extensionality principles. Agda designers point out in [Coc20] that having local rewrite rules would improve modularity. For example, one could parameterize code by an abstract type, together with rewrite rules it satisfies. This sort of functionality is already present in Andromeda 2, which treats all judgement forms as first-class values, so we may simply pass judgemental equalities as parameters and use them as local computation and extensionality rules.

In order to make our equality checking algorithm realistically useful, we ought to combine it with other techniques, such as existential variables, unification, and implicit arguments. Whether that can be done in full generality remains to be seen.

\section{REFERENCES}

[Agd] The Agda proof assistant. https://wiki.portal.chalmers.se/agda/.

[And] The Andromeda proof assistant. http://www.andromeda-prover.org/.

[AOV17] Andreas Abel, Joakim Öhman, and Andrea Vezzosi. Decidability of Conversion for Type Theory in Type Theory. Proceedings of the ACM on Programming Languages, 2(POPL), December 2017.

[AS12] Andreas Abel and Gabriel Scherer. On Irrelevance and Algorithmic Equality in Predicative Type Theory. Logical Methods in Computer Science, 8(1), 2012. 
[BGH ${ }^{+}$18] Andrej Bauer, Gaëtan Gilbert, Philipp G. Haselwarter, Matija Pretnar, and Christopher A. Stone. Design and Implementation of the Andromeda Proof Assistant. In 22nd International Conference on Types for Proofs and Programs (TYPES 2016), volume 97 of LIPIcs, pages 5:1-5:31, 2018.

[BHL20] Andrej Bauer, Philipp G. Haselwarter, and Peter LeFanu Lumsdaine. A general definition of dependent type theories. arXiv:2009.05539, September 2020.

[BHP20] Andrej Bauer, Philipp G. Haselwarter, and Anja Petković. Equality Checking for General Type Theories in Andromeda 2. In Mathematical Software - ICMS 2020, pages 253-259, 2020.

[CA16] Jesper Cockx and Andreas Abel. Sprinkles of extensionality for your vanilla type theory. In 22nd International Conference on Types for Proofs and Programs TYPES 2016, University of Novi Sad, 2016.

[Cha12] Arthur Charguéraud. The Locally Nameless Representation. Journal of Automated Reasoning, 49:363-408, 2012.

[Coc20] Jesper Cockx. Type Theory Unchained: Extending Agda with User-Defined Rewrite Rules. In Marc Bezem and Assia Mahboubi, editors, 25th International Conference on Types for Proofs and Programs (TYPES 2019), volume 175 of Leibniz International Proceedings in Informatics (LIPIcs), pages 2:1-2:27, Dagstuhl, Germany, 2020. Schloss Dagstuhl-Leibniz-Zentrum für Informatik.

[Coq] The Coq proof assistant. https://coq.inria.fr/.

[CTW21] Jesper Cockx, Nicolas Tabareau, and Théo Winterhalter. The Taming of the Rew: A Type Theory with Computational Assumptions. Proceedings of the ACM on Programming Languages, 5(POPL), 2021.

[Ded] The Dedukti logical framework. https://deducteam.github.io.

$\left[\mathrm{dMKA}^{+} 15\right]$ Leonardo de Moura, Soonho Kong, Jeremy Avigad, Floris van Doorn, and Jakob von Raumer. The Lean theorem prover (system description). In 25th International Conference on Automated Deduction (CADE 25), August 2015.

[GCST19] Gaëtan Gilbert, Jesper Cockx, Matthieu Sozeau, and Nicolas Tabareau. Definitional proofirrelevance without K. Proceedings of the ACM on Programming Languages, 3(POPL):3:1-3:28, 2019.

[HB21] Philipp G. Haselwarter and Andrej Bauer. Finitary type theories with and without contexts. arXiv:2112.00539, December 2021.

[MMT] The MMT Language and System. https://uniformal.github.io//.

[MP93] James McKinna and Robert Pollack. Pure Type Systems Formalized. In Mark Bezem and Jan F. Groote, editors, International Conference on Typed Lambda Calculi and Applications (TLCA), volume 664 of Lecture Notes in Computer Science. Springer, Berlin, Heidelberg, 1993.

[Rab18] Florian Rabe. A Modular Type Reconstruction Algorithm. ACM Transactions on Computational Logic, 19(4):24:1-24:43, 2018.

[SAG20] Jonathan Sterling, Carlo Angiuli, and Daniel Gratzer. A Cubical Language for Bishop Sets. arXiv:2003.01491, March 2020.

$\left[\mathrm{SBF}^{+} 19\right]$ Matthieu Sozeau, Simon Boulier, Yannick Forster, Nicolas Tabareau, and Théo Winterhalter. Coq Coq correct! Verification of type checking and erasure for Coq, in Coq. Proceedings of the ACM on Programming Languages, 4(POPL), December 2019.

[SH06] Christopher A. Stone and Robert Harper. Extensional equivalence and singleton types. $A C M$ Transactions on Computational Logic, 7(4):676-722, 2006. 